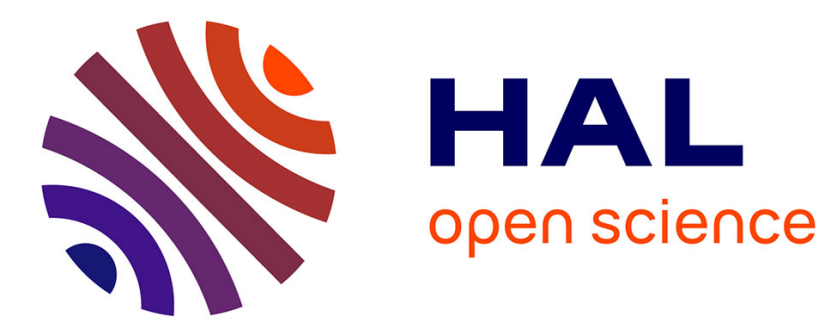

\title{
Absorbing boundary conditions for Rayleigh waves
}

\author{
A. Bamberger, Patrick Joly, Jean Roberts, J.L. Teron
}

\section{To cite this version:}

A. Bamberger, Patrick Joly, Jean Roberts, J.L. Teron. Absorbing boundary conditions for Rayleigh waves. [Research Report] RR-0384, INRIA. 1985. inria-00076172

\section{HAL Id: inria-00076172 \\ https://hal.inria.fr/inria-00076172}

Submitted on 24 May 2006

HAL is a multi-disciplinary open access archive for the deposit and dissemination of scientific research documents, whether they are published or not. The documents may come from teaching and research institutions in France or abroad, or from public or private research centers.
L'archive ouverte pluridisciplinaire HAL, est destinée au dépôt et à la diffusion de documents scientifiques de niveau recherche, publiés ou non, émanant des établissements d'enseignement et de recherche français ou étrangers, des laboratoires publics ou privés. 


\title{
ABSORBING BOUNDARY CONDITIONS \\ FOR RAYLEIGH WAVES
}

\begin{abstract}
Alain BAMBERGER ${ }^{*}$, Patrick JOLY*, Jean Elizabeth ROBERTS ${ }^{*}$, Jean Luc TERON ${ }^{* *}$.
\end{abstract}

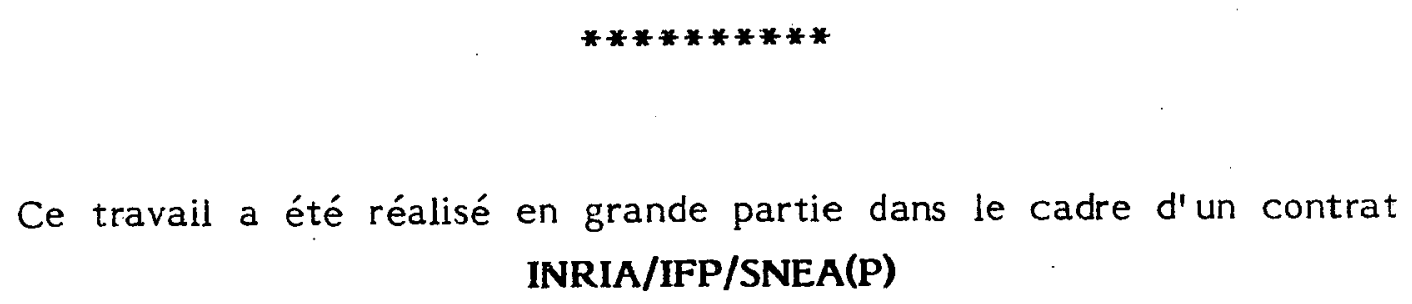

INRIA/IFP/SNEA(P) 


\section{ABSTRACT}

The first order absorbing boundary conditions for elastic waves are transparent for $P$ waves and $S$ waves at normal incidence but give rise to parasitic reflections of Rayleigh waves. To treat this phenomena we first propose a solution of geometric type which eliminates these parasitic waves but causes others to appear which, while less important, are. still troublesome. We propose a second solution by constructing a new condition of second order type, transparent for $P$ waves and $s$ waves at normal incidence and also for Rayleigh waves. We analyse this condition mathematically and demonstrate its good behavior with regard to reflection phenomena.

\section{RESUME}

Les conditions absorbantes $\mathrm{du} 1^{e r}$ ordre pour les ondes élastiques sont transparentes pour les ondes $P$ et $S$ à incidence normale mais font apparaitre des réflexions $d^{\prime}$ ondes de Rayleigh parasites.

Pour éliminer ces phénomènes, nous proposons une première solution de type géométrique, qui permet de réduire leur amplitude mais en fait naitre de nouveaux, de moindre importance (quoique toujours génants).

Nous proposons une seconde solution en construisant une nouvelie condition, type second ordre, transparente pour les ondes $P$ et $S \grave{a}$ incidence normale et pour les ondes de Rayleigh. Nous analysons mathématiquement cette condition et mettons en évidence ses bonnes propriétés vis à vis des phénomènes de réflexion.

\section{KEY WORDS}

Absorbing boundary conditions, elastodynamics, Rayleigh waves.

\section{MOT CLES}

Conditions aux bords absorbantes, ondes élastiques, ondes de Rayleiğt. 


\section{I - REFLECTION OF RAYLEIGH WAVES BY THE FIRST}

\section{ORDER CONDITION}

\section{I.1 - The first order absorbing boundary condition and its properties}

We recall that the first order absorbing boundary condition for the equations of elastodynamics is

$$
\sigma \cdot n+M \frac{\partial u}{\partial t}=0
$$

at each point of the absorbing boundary with exterior normal $n=\left(n_{1}, n_{2}\right)$, where $M$ is the matrix given by :

$$
M=\left(\begin{array}{cc}
n_{1} & n_{2} \\
n_{2} & -n_{1}
\end{array}\right)\left(\begin{array}{cc}
\rho V_{P} & 0 \\
0 & \rho v_{S}
\end{array}\right)\left(\begin{array}{cc}
n_{1} & n_{2} \\
n_{2} & -n_{1}
\end{array}\right) .
$$

Note that the matrix $M$ is symmetric and positive definite and thus the boundary condition expresses the fact that at the boundary the normal stress on $\Gamma$ is inversely proportional to the velocity.

By construction, the condition $((1.1),(1.2))$ is the unique condition of type (1.1), with $M$ symmetric and positive definite, which is transparent for all plane harmonic $P$ and $S$ waves arriving at $\Gamma$ with normal incidence. Note also that for each displacement field $u$ satisfying the equations of elastodynamics in $\Omega$ and $a$ condition of type (1.1) on $\Gamma$, with $M$ symmetric and positive definite, we have the energy identity :

(1.3) $\quad \frac{1}{2} \frac{d}{d t}\{E(\Omega, t)\}+\int_{\Gamma} M \frac{\partial u}{\partial t} \cdot \frac{\partial u}{\partial t} d \sigma=0$, 
where $E(\Omega, t)$ denotes the total energy of the field $u$ contained at instant $t$ in the domain $\Omega$.

The equality (1.3) implies, in particular, that the total energy is a nonincreasing function of time, i.e. any boundary condition of type (1.1) is an absorbing condition and thus leads to a well posed mathematical problem.

We summarize these well known properties in the following theorem.

\section{Theorem 1.1}

Formula (1.1), where $M$ is a symmetric, positive definite matrix, defines a family of absorbing boundary conditions for the equations of elastodynamies in two dimensions. If $M$ is given by (1.2) the resulting boundary condition is the unique condition of this family which is transparent for all plane harmonic $P$ and $S$ waves striking the boundary $\Gamma$ with normal incidence.

We remark that the first order condition (1.1), (1.2) depends on the geometry of the domain $\Omega$ since $M$ depends on the unit exterior normal n.

In particular this conditions becomes :

- in the case of a vertical boundary $\left(x_{1}=0\right)$

$$
\left(\begin{array}{c}
\sigma_{11} \\
\sigma_{21}
\end{array}\right)+\rho\left(\begin{array}{cc}
v_{P} & 0 \\
0 & v_{S}
\end{array}\right)\left(\begin{array}{c}
\frac{\partial u_{1}}{\partial t} \\
\frac{\partial u_{2}}{\partial t}
\end{array}\right)=0
$$

- in the case of a horizontal boundary $\left(x_{2}=0\right)$

$$
\left(\begin{array}{l}
\sigma_{12} \\
\sigma_{22}
\end{array}\right)+\rho\left(\begin{array}{cc}
v_{S} & 0 \\
0 & v_{P}
\end{array}\right)\left(\begin{array}{c}
\frac{\partial u_{1}}{\partial t} \\
\frac{\partial u_{2}}{\partial t}
\end{array}\right)=0 .
$$


Classically, the first order condition $((1.1),(1.2))$ is analyzed in terms of reflection of a plane harmonic $P$ or $S$ wave, with amplitude 1 , striking the absorbing boundary $\Gamma$ with an incidence $\theta$, $0 \leqq \theta \leqq \pi / 2$. One can show that, for a given $\theta$ :

- An incident $P$ wave gives rise to a reflected $P$ wave with amplitude $R_{P P}(\theta, \nu)$ and to a reflected $S$ wave of amplitude $R_{P S}(\theta, \nu)$ - An incident $S$ wave gives rise to a reflected $S$ wave with amplitude $R_{S S}(\theta, \nu)$ and to a reflected $P$ wave of amplitude $R_{S P}(\theta, \nu)$. The coefficients $R_{P P}(\theta, \nu), R_{P S}(\theta, \nu)$ (resp. $R_{S S}(\theta, \nu)$, $\left.R_{S P}(\theta, v)\right)$ are by definition the reflection coefficients of a $P$ wave (resp. of an $S$ wave) for the absorbing boundary $\Gamma$. They depend only on the angle of incidence $\theta$ and the poisson coefficient $\nu$.

In particular one can show that for small angles of incidence $\theta$ that :

$$
\left\{\begin{array}{l}
\mathrm{R}_{\mathrm{PP}}(\theta, \nu)=O\left(\theta^{2}\right) \\
\mathrm{R}_{\mathrm{PS}}(\theta, \nu)=O(\theta) \\
\mathrm{R}_{\mathrm{SS}}(\theta, \nu)=O\left(\theta^{2}\right) \\
\mathrm{R}_{\mathrm{SP}}(\theta, \nu)=O(\theta),
\end{array}\right.
$$

which shows that the first order condition is "quasi-transparent" for small values of $\theta$, the results being in each case better for the phenomena of pure reflection $(P \rightarrow P, S \rightarrow S)$ than for the phenomena of conversion $(P \rightarrow S, S \rightarrow P)$. 


\section{I.2 - The reflection of Aayleigh waves}

We consider now the numerical simulation of the propagation of elastic waves in a homogeneous half space, $x_{2} \leq 0$, with the free surface boudary condition on the boundary $\Gamma_{1}\left(x_{2}=0\right)$,

$(1.4) \quad\left(\begin{array}{c}\sigma_{12} \\ \sigma_{22}\end{array}\right)=0 \quad$ on $\Gamma_{1} \cdot$

It is well known that in such a medium besides the so called "volume waves", i.e. $P$ and $S$ waves, there also propagate surface waves guided by the free boundary $\Gamma_{1}$. These are the Rayleigh waves which are the solutions of the form

$$
u_{R}\left(x_{1}, x_{2}, t\right)=u_{R}\left(x_{1} \pm v_{R} t, x_{2}\right)
$$

where $C=V_{R}$ is the unique solution in the interval $\left[0, V_{S}\right]$ of the classical Rayleigh equation

$$
4\left(1-\frac{C^{2}}{V_{P}^{2}}\right)^{1 / 2}\left(1-\frac{C^{2}}{V_{S}^{2}}\right)^{1 / 2}=\left(2-\frac{C^{2}}{V_{S}^{2}}\right)^{2}
$$

For the present we shall limit our domain of calculation to the quarter plane

$$
\Omega=\left\{\left(x_{1}, x_{2}\right) \in \mathbb{R}^{2}: x_{1} \leqq 0, x_{2} \leqq 0\right\},
$$

and take for boundary condition on the artificial boundary $\Gamma_{2}\left(x_{1}=0\right)$ the first order absorbing boundary condition,

$$
\left(\begin{array}{c}
\sigma_{11} \\
\sigma_{21}
\end{array}\right)+\rho\left(\begin{array}{cc}
v_{P} & 0 \\
0 & v_{S}
\end{array}\right)\left(\begin{array}{c}
\frac{\partial u_{1}}{\partial t} \\
\frac{\partial u_{2}}{\partial t}
\end{array}\right)=0 \text { on } \Gamma_{1} \cdot
$$




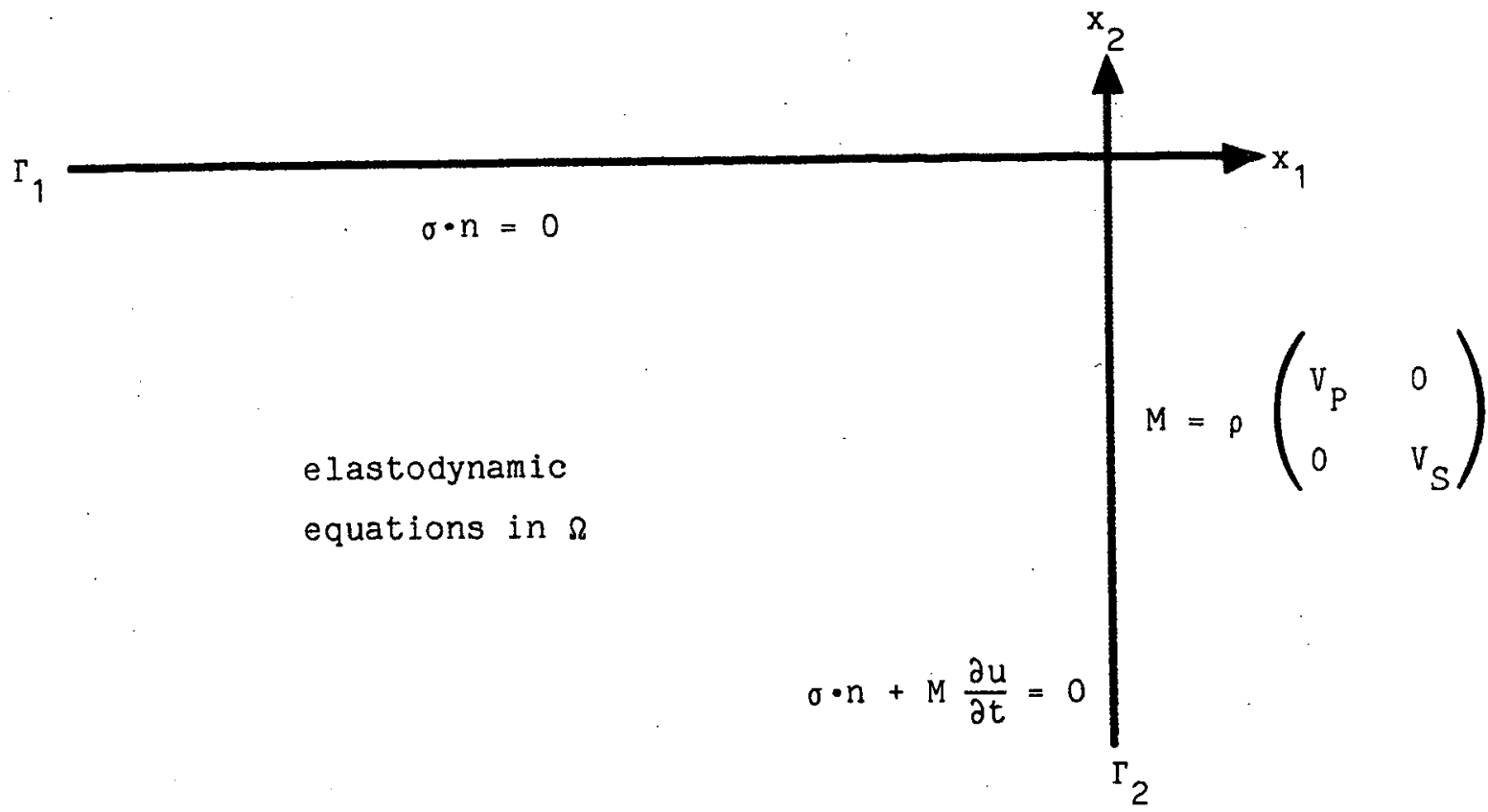

One can easily verify that a Rayleigh wave propagating along the free boundary $\Gamma_{1}$ in the positive $x_{1}$ direction, i.e. a wave incident upon the artificial boundary $\Gamma_{2}$,

$$
u\left(x_{1}, x_{2}, t\right)=U_{R}\left(x_{1}-v_{R} t, x_{2}\right),
$$

does not satisfy condition (1.7). In other words, the first order condition is not transparent for Rayleigh waves.

Thus, the question that naturally arises is the following : what happens when a Rayleigh wave reaches the absorbing boundary $\mathrm{I}_{2}$ ? The mathematical analysis of the phenomenon is extremely difficult. only a numerical simulation permits us to have, in a simple manner, an idea of what happens, at least qualitatively ... 
The results we shall present now were obtained by limiting the domain of calculation to a rectangle $\Omega$. One of the sides of the rectangle coincides with the free boundary $\Gamma_{1}$. The three other sides are absorbing; we have taken the first order condition.

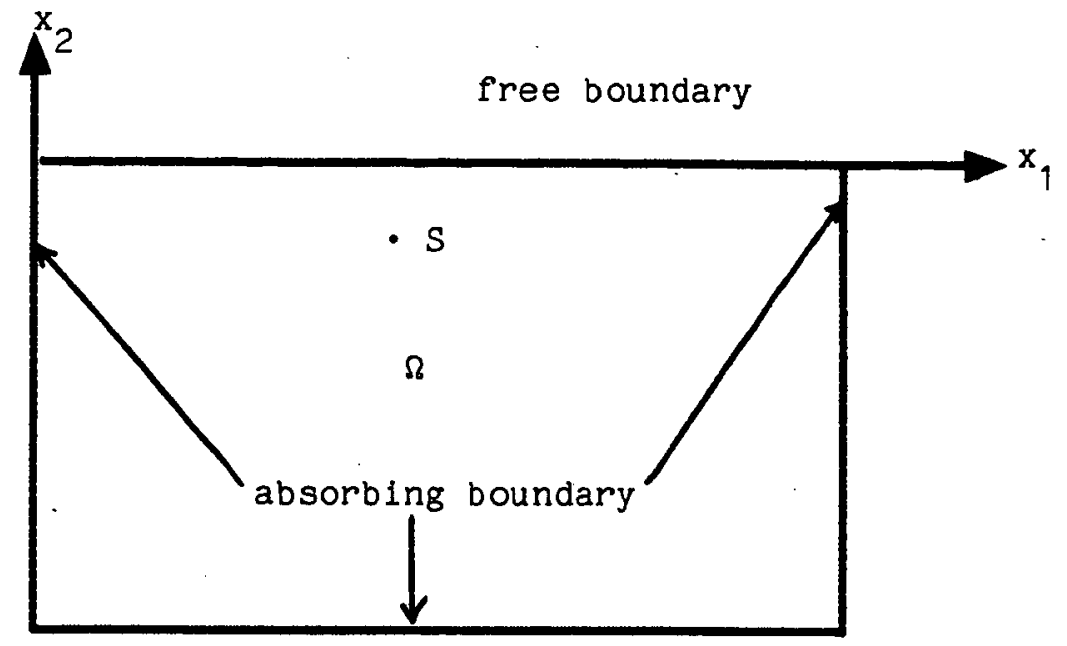

The elastic waves are excited by the explosion, at time $t=0$, of a seismic source located at point $S$ near the free surface. The following results were obtained by a numerical simulation in which $Q 1$ finite elements associated with a uniform grid were used for the spatial discretization, and an explicit scheme was employed for the time discretization.

Figures 1.1 through 1.5 represent "snapshots!" of the elastic medium $\Omega$ at different instants. Each figure gives, at a given time, an image of the deformed medium, in that each point of the grid has been displaced by its (amplified) displacement vector $u$. Thus the compressed regions are darker and the dilated regions lighter.

The source was placed at the surface in order to generate surface waves with a large amplitude. We consider a source of compressional waves. The $P$ wave front we see only in figure 1.1 . 


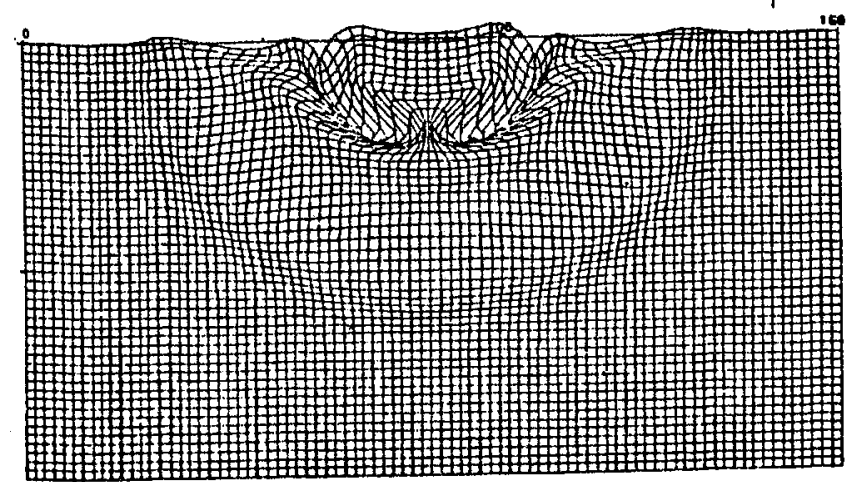

Figure 1.1:

Deformed grid

Date : 40 milliseconds

Amplification Factor

$(\mathrm{AF})=3 \times 10^{-2}$

one trace every 2 meters.

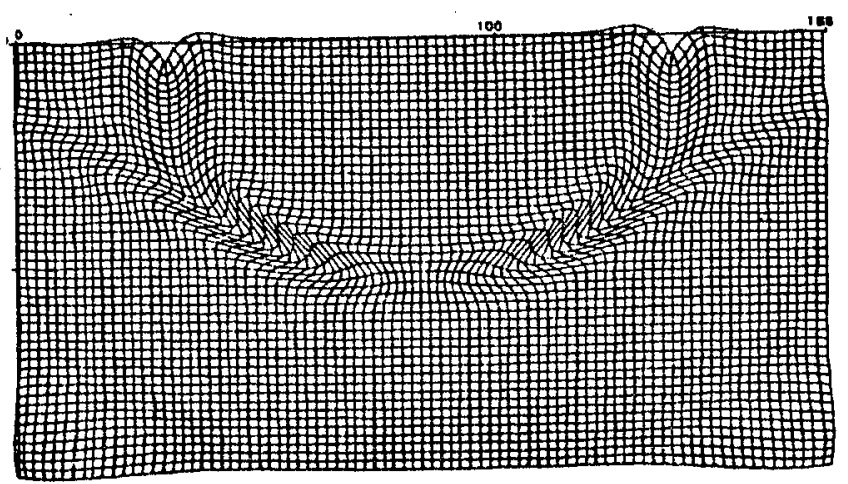

Figure 1.2:

Deformed grid

Date : 80 milliseconds

$\mathrm{AF}=3 \times 10^{-2}$

One trace every 2 meters

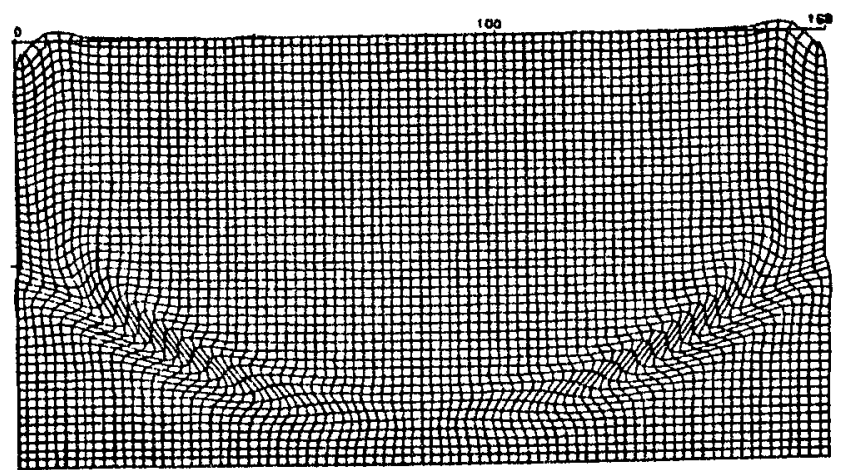

Figure 1.3:

Deformed grid

Date : 120 milliseconds

$A F=3 \times 10^{-2}$

One trace every two meters

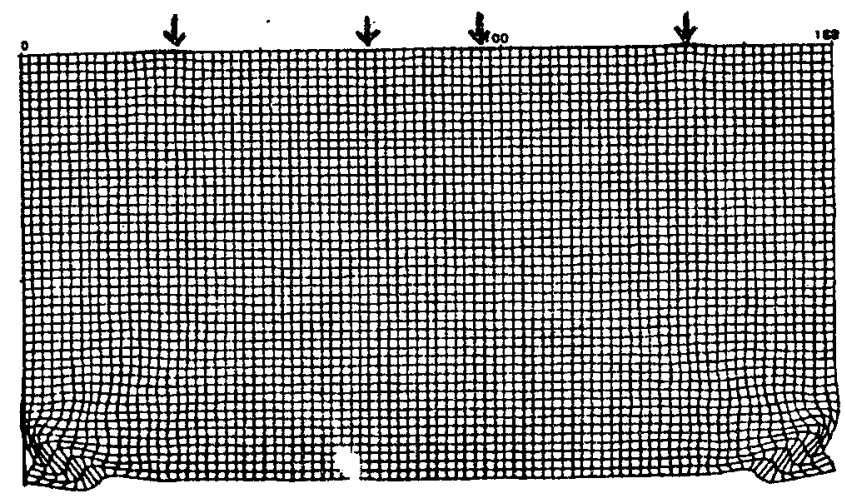

Figure $1.4:$

Deformed grid

Date $160 \mathrm{milliseconds}$

$\mathrm{AF}=3 \times 10^{-2}$

One trace every two meters

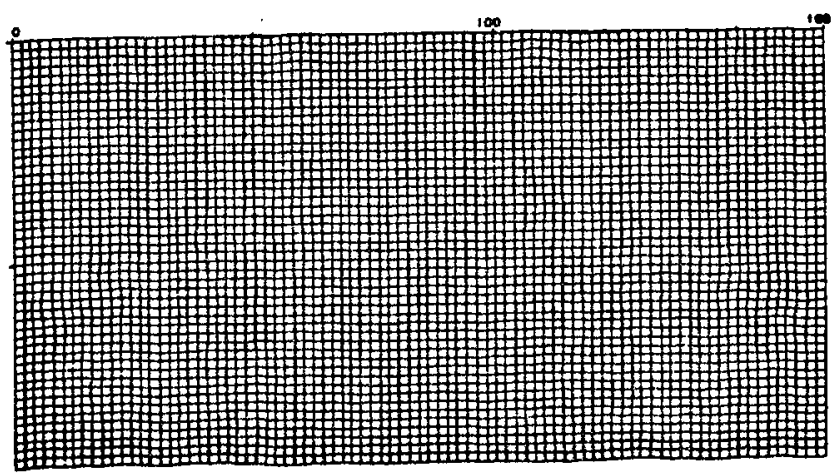

Figure 1.5:

Deformed grid

Date : 200 milliseconds

$\mathrm{AF}=3 \times 10^{-2}$

one trace every two meters. 
The second circular front is due to the conversion at the free surface of the $P$ wave to an $S$ wave. This shear wave is joined to the $P$ wave front by the head wave and to the Rayleigh wave, which corresponds to the large perturbation which disturbs the free surface as it propagates along it.

In. figures 1.1 and 1.2 , the Rayleigh wave has not yet reached the absorbing boundary. On the other hand, we can see that the $P$ and $S$ waves cross the artificial boundary without being noticeably reflected. The Rayleigh wave reaches the vertical boundary in figure 1.3 , and in figures 1.4 and 1.5 we see that it has suffered a parasitic reflection. One can observe, propagating along the surface, two fronts converging toward the source, one with velocity $V_{P}$ and the other with velocity $V_{R}$. While these parasitic waves might appear to be negligible, in fact they are not at all : the waves are of the same order of magnitude as the quantities actually measured in seismic reflection experiments which are themselves due to the reflection of the emitted wave by the heterogeneities of the substratum.

A finer analysis of these phenomena can be carried out by the examination of synthetic seismograms, which, placed one next to the other, are the diagrams depicting the curves that give the variations of the two components, vertical and horizontal, of the displacements of the points of the free surface as functions of time. As the amplitude of most of the waves decreases with time, we have amplified the results linearly in time in order to better observe the parasitic phenomena, see figures 1.6 and 1.7 .

one can clearly distinguish two lines $D_{p}$ and $D_{R}$ with respective slopes $\frac{1}{V_{p}}$ and $\frac{1}{V_{R}}$ which correspond to the propagation of the $P$ wave and the Rayleigh wave. Equally well one sees lines, $D_{R P}$ and $D_{R R}$, issuing from the point of intersection of the line $D_{R}$ with the curve corresponding to the right extremity of the model. These lines have slopes $-\frac{1}{V_{p}}$ and $-\frac{1}{V_{R}}$ respectively. 
$-9-$

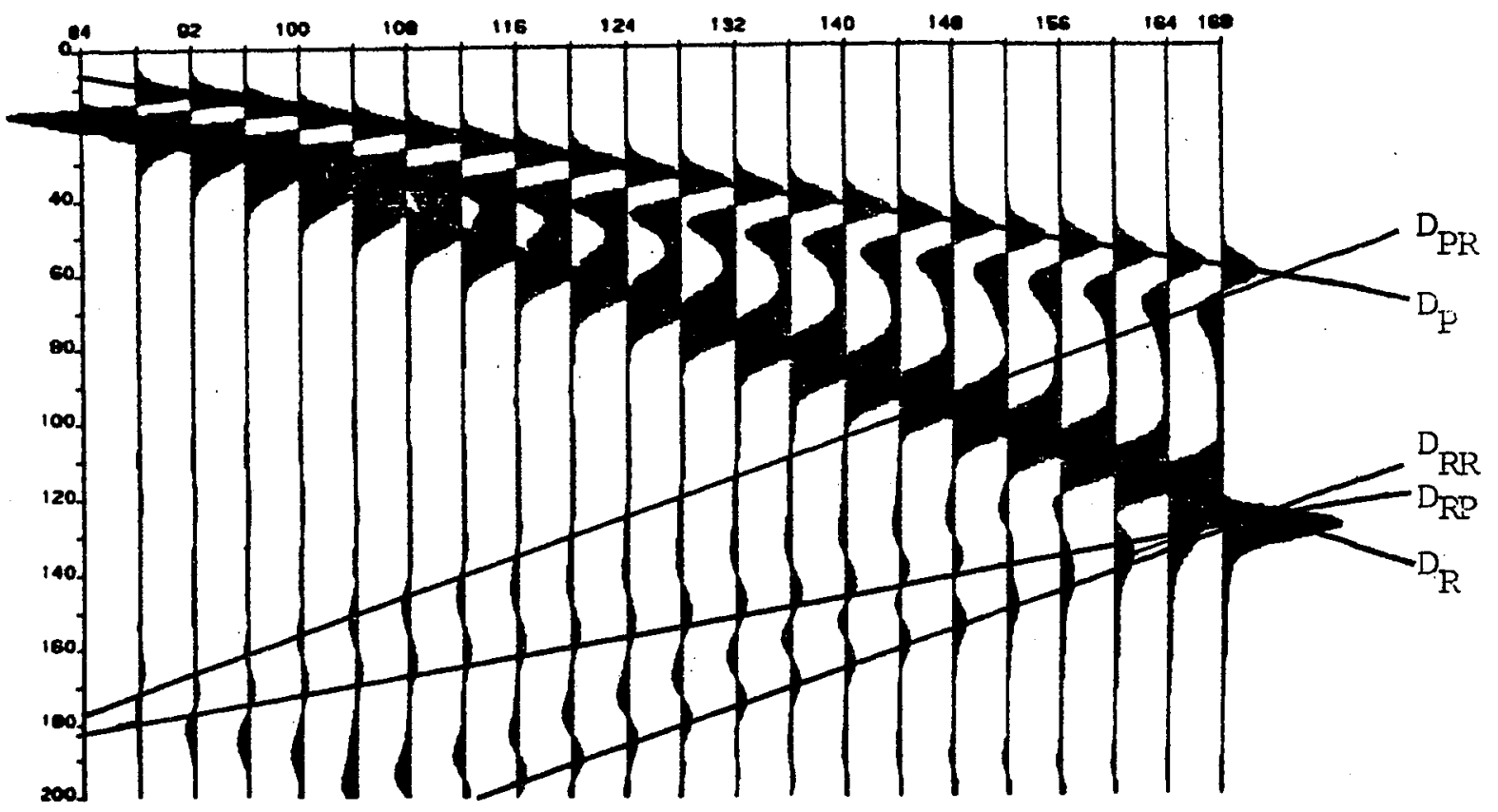

Figure 1.6:

Horizontal displacement

Initial Amplification Factor (IAF) $=1 \times 10^{-2}$

Gradient of Amplification (GA) $=1 \times 10^{-4}$

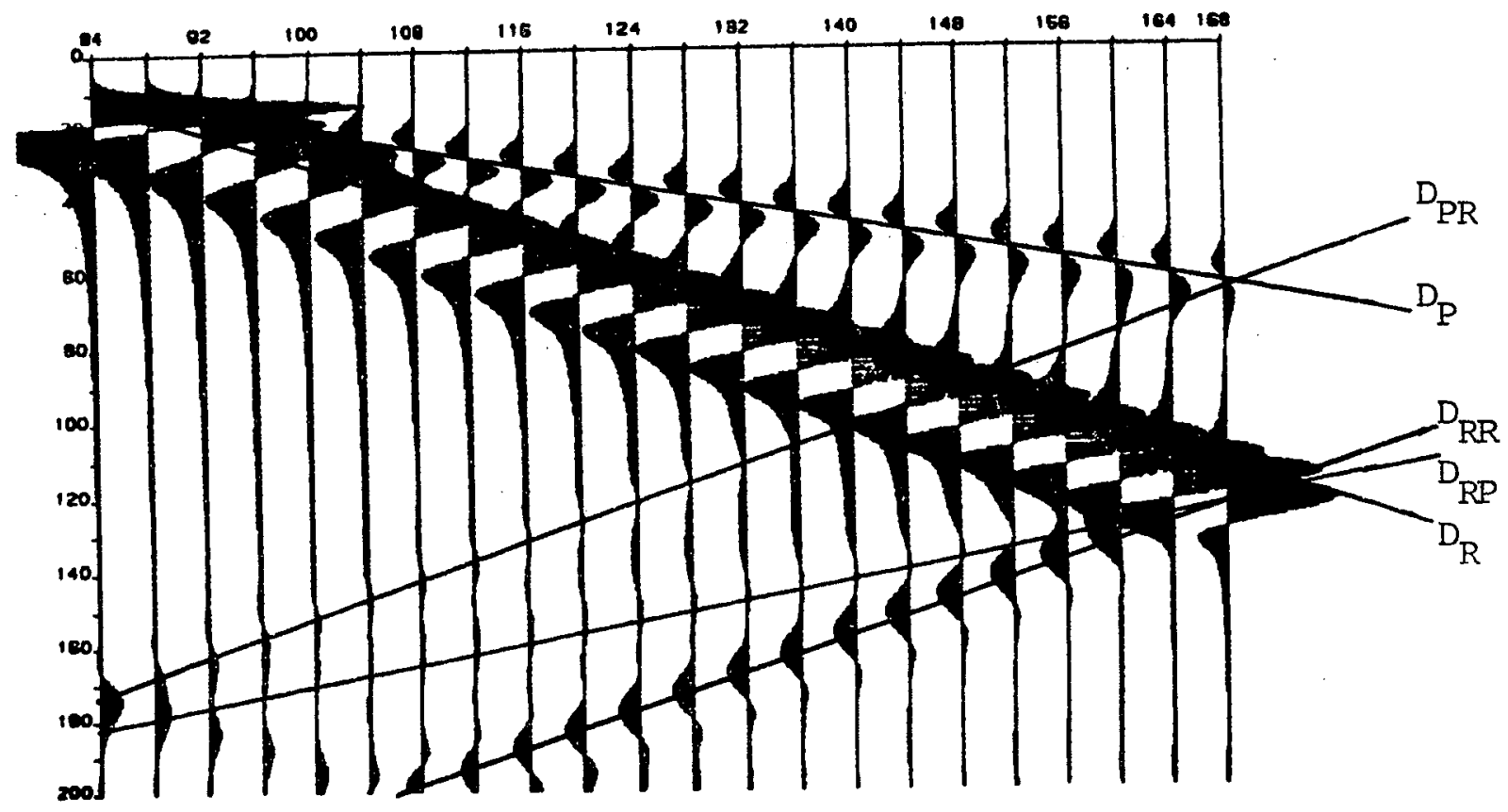

Figure $1.7:$

$$
\begin{aligned}
& \text { Vertical displacement } \\
& \mathrm{IAF}=1 \times 10^{-2} \\
& \mathrm{GA}=1 \times 10^{-4}
\end{aligned}
$$


The line $\quad D_{R R}$ with slope $-\frac{1}{V_{R}}$ corresponds to the Rayleigh wave reflected by the absorbing boundary. Arriving at the vertical boundary, the incident Rayleigh wave also gives rise to a compression wave which seems to be emitted by the corner point where the free and artificial boundaries intersect. It is this wave that explains the presence of the line $D_{R P}$ with slope $-\frac{1}{V_{P}}$. This is a phenomenon of conversion from a surface wave to a volume wave. These two lines correspond, of course, to the parasitic fronts observed in figure 1.4. There appears yet another line $D_{P R}$, a second line of slope $-\frac{1}{V_{R}}$, which intersects the rightmost seismogram, the one corresponding $^{\mathrm{R}}$ to the registration at the upper, right corner of the model, at the same point as does the line $D_{P}$ representing the incident $P$ wave: this $P$ wave, arriving at the vertical boundary, generates a parasitic Rayleigh wave. Anyway, this wave has a very small amplitude, even when compared with that of the reflected Rayleigh wave represented by the line $D_{R R}$, and is visible in figures 1.6 and 1.7 only for large values of $t$, i.e. when the amplification factor is maximum. This is a phenomenon of conversion of a volume wave to a surface wave. This is not in contradiction with the fact that the first order absorbing condition is transparent for normally incident $P$ waves as here we have, in fact, the presence of a corner joining a free boundary with an absorbing boundary, a condition that we do not know how to analyse mathematically. 


\section{II - A FIRST SOLUTION OF GEOMETRIC TYPE: EARS}

In the previous section we have seen that though the first order absorbing boundary condition is transparent for normally incident $P$ and $S$ waves, when this boundary condition is used for an artificial boundary in the problem of a half space with free boundary, we still have the problem of reflected and converted surface waves. There are two evident ways to approach this problem : The first, and more rudimentary, is to try to modify the geometry of the domain of calculation to avoid having the corner connecting the free bondary with the absorbing boundary. The second is to modify the boundary condition itself. It is the first of these that we shall consider in this paragraph.

Since the amplitude of Rayleigh waves decreases exponentially with the distance from the free boundary, a very simple solution to the problem of the reflection of such waves at an artificial boundary is to enlarge the domain of calculation in a small region around the free boundary. In this way the. length of time necessary for a Rayleigh wave to reach the artificial boundary and to be reflected back into the domain of interest can be made larger than the length of time of observation so that no reflection of a Rayleigh wave is detected.

Thus if we consider the example of the previous paragraph, the idea is to modify our rectangular domain of calculation by adding to it two long thin rectangles (ears) in such a way as to extend the length of the free boundary on both sides of the original domain. More precisely, if we assume that our free boundary coincides with the axis $x_{2}=0$ and that our original domain was the rectangle $\Omega_{1}=[0,2 \ell] \times[0, d]$ with source $S$ at $(l, 0)$, then our enlarged domain would be :

$$
\Omega_{2}=\Omega_{1} \cup E_{\ell} \cup E_{r}
$$

where we have set (with $b<d$ )

$$
\left\{\begin{array}{l}
E_{\ell}=[-a, 0] \times[0, b] . \\
E_{r}=[2 \ell, 2 \ell+a] \times[0, b] .
\end{array}\right.
$$




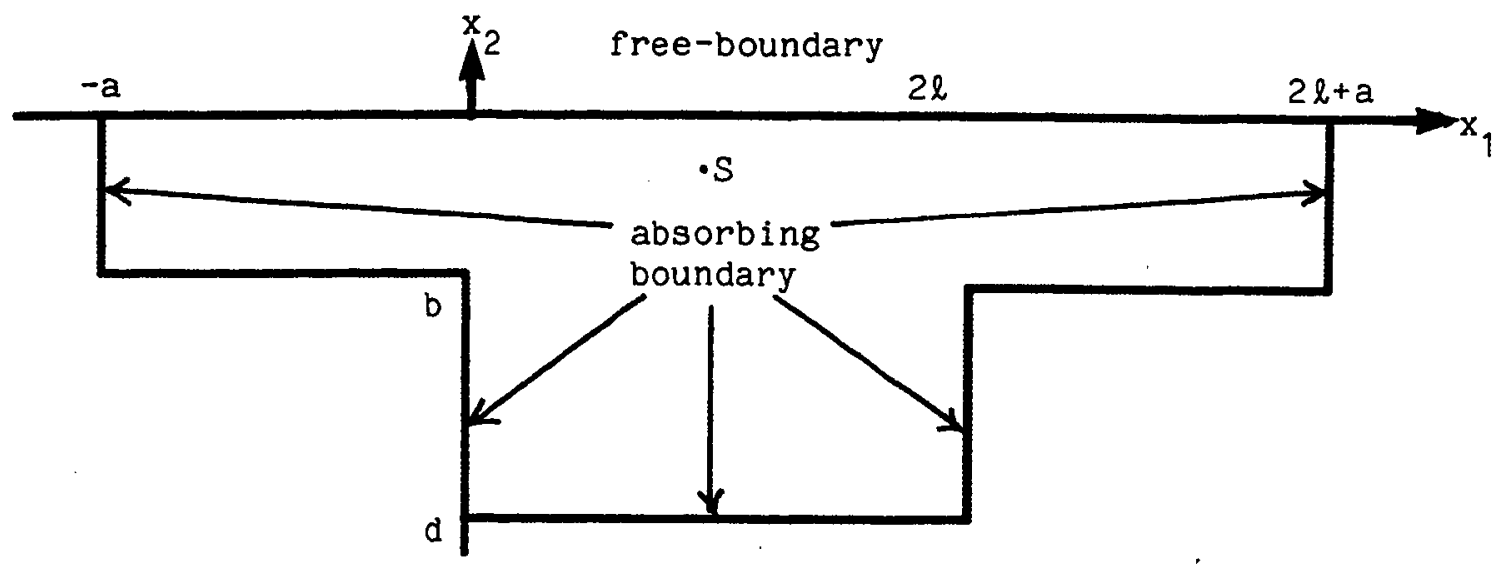

The constant a should be chosen large enough so that the time, $(\ell+a) / V_{R}+a / V_{P}$, needed for a Rayleigh wave to travel the distance $\ell+a$ and for the converted $P$ wave to return the distance a to the domain of observation exceeds $T$, where $T$ represents the final time of the observation. The depth $b$ should be just of sufficient size for the amplitude of a Rayleigh wave to be negligible at that distance from the free boundary, but not so large as to greatly increase the time of calculation.

The following figures represent the results of the same experiment as do those presented in paragraph 1, except that the domain of calculation here is $\Omega_{2}$ instead of $\Omega_{1}$. Here $\Omega_{1}$ is a rectangle of width $168 \mathrm{~m}$ and depth $96 \mathrm{~m}$ with source located in the center at depth 0 . We have taken the ear to be of depth $49 \mathrm{~m}$ though in practice the ratio of the depth of the ear to that of $\Omega_{1}$ would be much smaller.

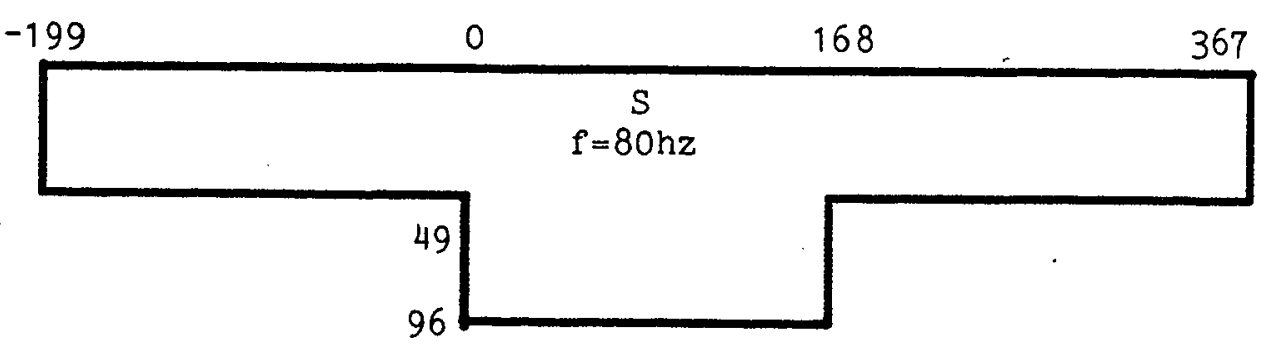




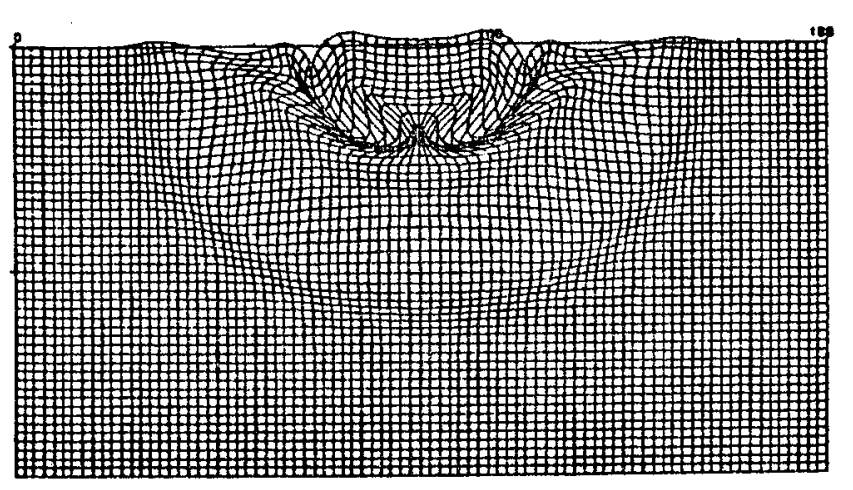

Figure 2.1 :

Deformed grid

Date : 40 milliseconds

Amplification Factor

$(\mathrm{AF})=3 \times 10^{-2}$

one trace every 2 meters

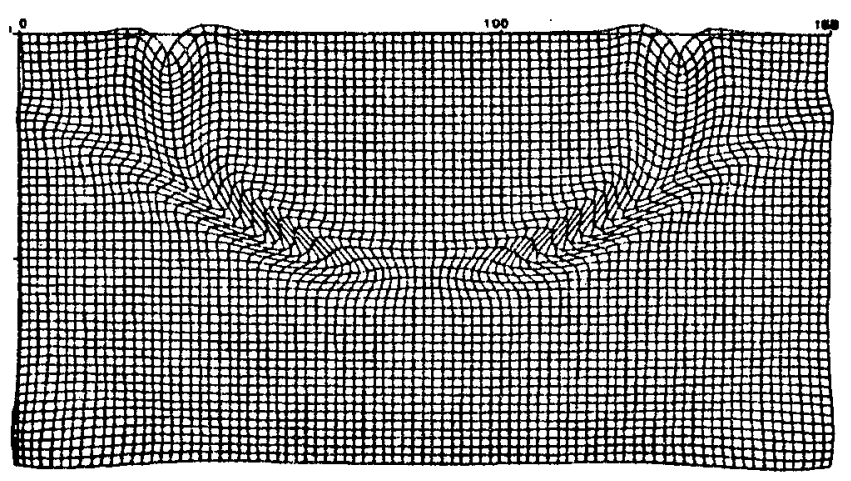

Figure 2.2:

Deformed grid

Date : $80 \mathrm{milliseconds}$

$\mathrm{AF}=3 \times 10^{-2}$

One trace every 2 meters

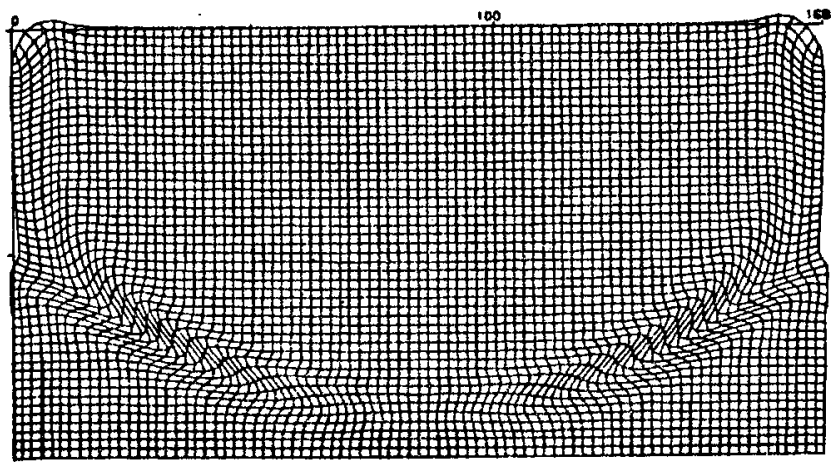

Figure 2.3:

Deformed grid

Date : 120 milliseconds

$\mathrm{AF}=3 \times 10^{-2}$.

one trace every 2 meters

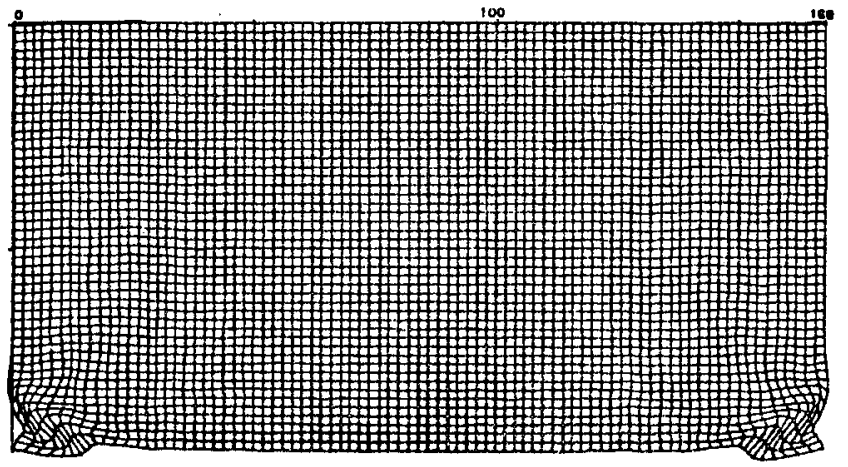

Figure 2.4:

Deformed grid

Date : $160 \mathrm{milliseconds}$

$A F=3 \times 10^{-2}$

one trace every 2 meters 
Figures 2.1 through 2.4 are the snapshots corresponding to those of figures 1.1 through 1.4. Figures 2.1 and 1.1 are, of course, identical as the disturbance has not yet reached the artificial boundary. However, the fact that 2.2 and 1.2 as well as 2.3 and 1.3 appear indistinguishable indicates that the $P$ wave and the $S$ wave fronts have passed the interior corners, located at $(0, b)$ and $(2 l, b)$ without any noticeable diffraction. In figure 2.3 the Rayleigh wave reaches the bounds of the observed domain and in figure 2.4 we see that it has passed into the ears without giving rise to the parasitic $P$ wave or Rayleigh wave observed in figure 1.4.

In figures 2.5 through 2.7 we restrict our attention to roughly the right quarter of the observed domain as well as to the right ear itself. The times of observation are the same as those in $1.2,1.3$, and 1.4 respectively as well as in $2.2,2.3$, and 2.4 . In figures $2.5,2.6$, and 2.7 , we see the Rayleigh wave and $S$ wave as they propagate toward the artificial boundary, reach the right ear, and move in to the ear with apparently no problem. However, to more closely observe what happens at the interior corner, we look again in figure 2.8 at the deformation seen in 2.7 but this time with an amplification factor 10 times larger. In figure 2.8 a diffraction due to the corner is definitely visible, though it is not immediately evident if this is due to the $S$ wave, the Rayleigh wave, or even the connecting wave between the two.

The seismograms corresponding to those presented in figures 1.6 and 1.7 are given in 2.9 and 2.10. As in 1.6 and 1.7 we can clearly distinguish the two lines $D_{p}$ and $D_{R}$, having slopes $1 / V_{p}$ and $1 / V_{R}$ respectively, resulting from the disturbance caused by the $P$ wave and Rayleigh wave arising at the source. However, the lines $D_{R R}$ and $D_{R P}$ which correspond to the reflected and converted Rayleigh wave as well as the line $D_{P R}$ corresponding to the conversion of the incident $P$ wave to a parasitic Rayleigh wave have disappeared. Thus, even for the large amplication factors toward the final time, there is no trace of these parasitic waves since the boundary giving rise to them has been pushed further from the source. 

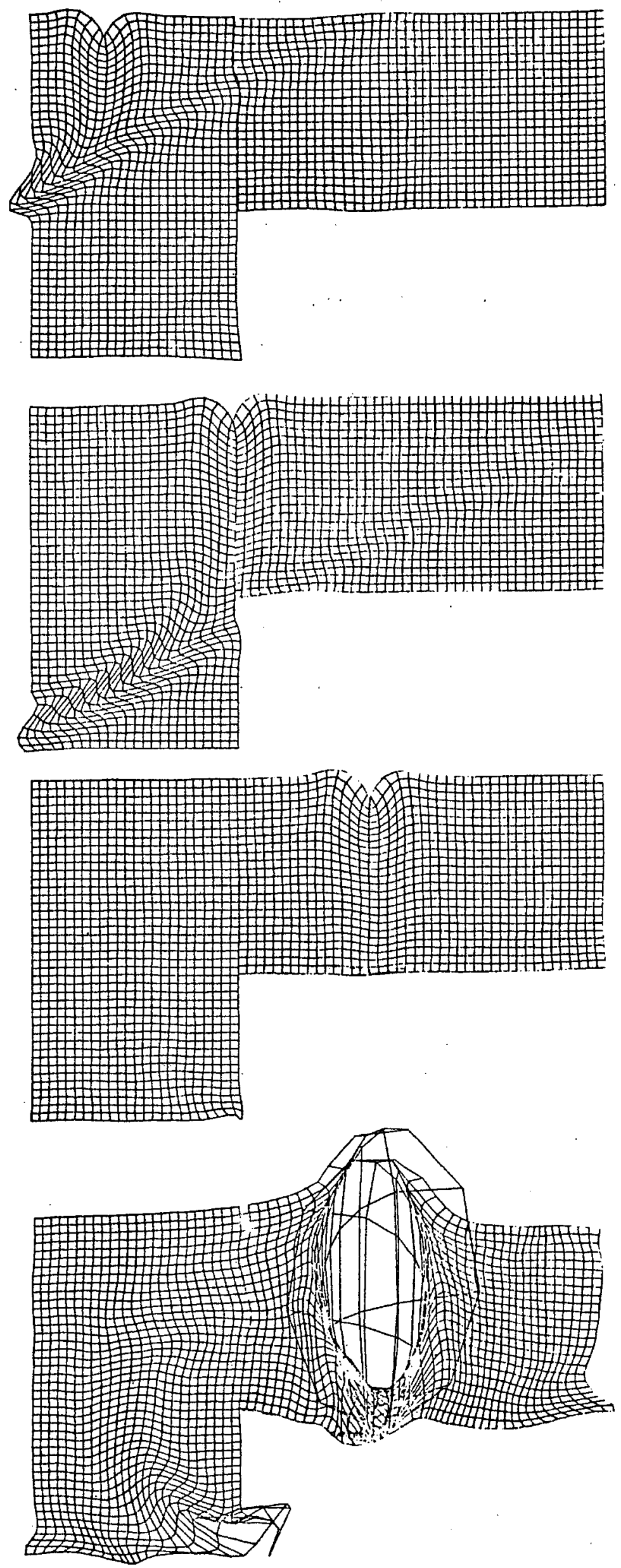

Figure 2.5 :

Deformed grid

Date : 80 milliseconds

$A F=3 \times 10^{-2}$

One trace every 2 meters

Figure 2.6 :

Deformed grid

Date : $120 \mathrm{milliseconds}$

$\mathrm{AF}=3 \times 10^{-2}$

One trace every 2 meters

Figure 2.7:

Deformed grid

Date : 160 milliseconds

$\mathrm{AF}=3 \times 10^{-2}$

One trace every 2 meters

Figure 2.8:

Deformed grid

Date : 160 milliseconds

$\mathrm{AF}=3 \times 10^{-2}$

One trace every 2 meters 


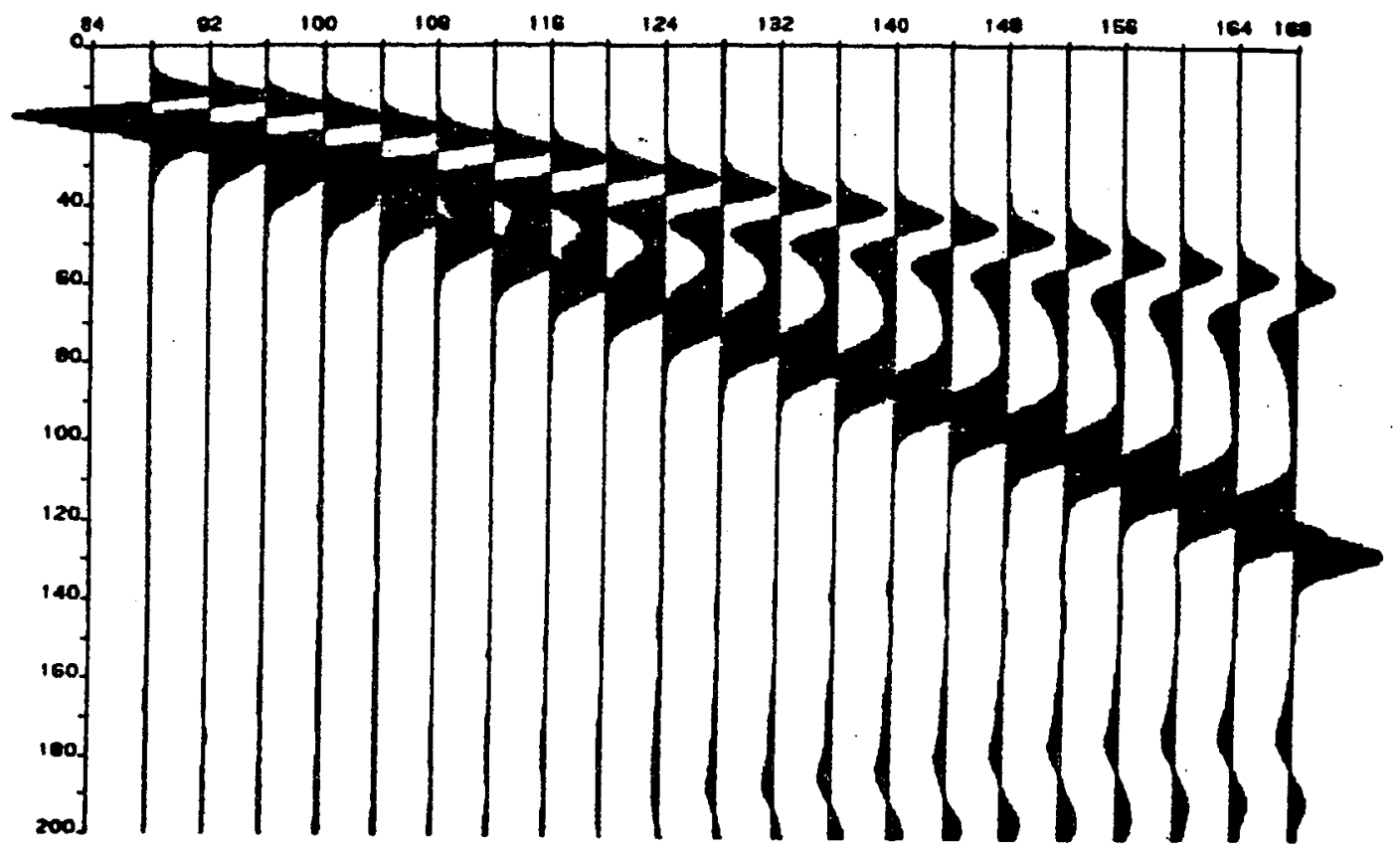

Figure 2.9: Horizontal Displacement

Initial Amplification Factor (IAF) $=1 \times 10^{-2}$

Gradient of Amplification $(G A)=1 \times 10^{-4}$

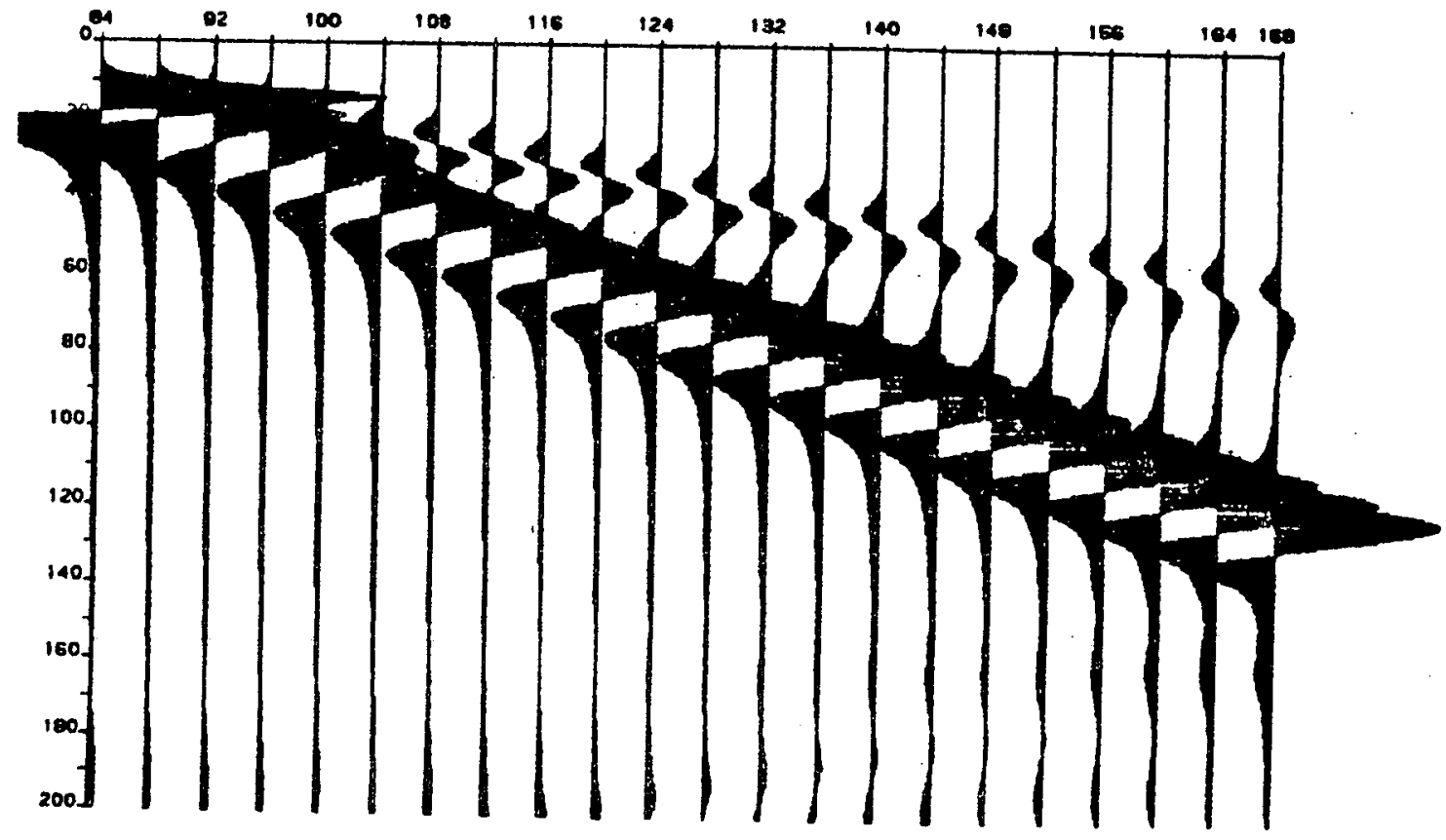

Figure 2.10: Vertical Displacement

$I F A=1 \times 10^{-2}$

$\mathrm{GA}=1 \times 10^{-4}$ 
However, in figure 2.9 , representing horizontal displacement, and to a lesser extent in figure 2.10 representing vertical displacement, there is a definite disturbance indicated in the lower righthand corner. This perturbation corresponds to the phenomenon already detected in the snapshot 2.8, which is due to the diffraction at the interior corner.

To further examine the phenomena of diffraction at the interior corner we have repeated the previous experiment increasing the depth of the original rectangle to 192 meters and that of the ears to 99 meters and also increasing the final time of the observation from 200 milliseconds to 400 milliseconds.

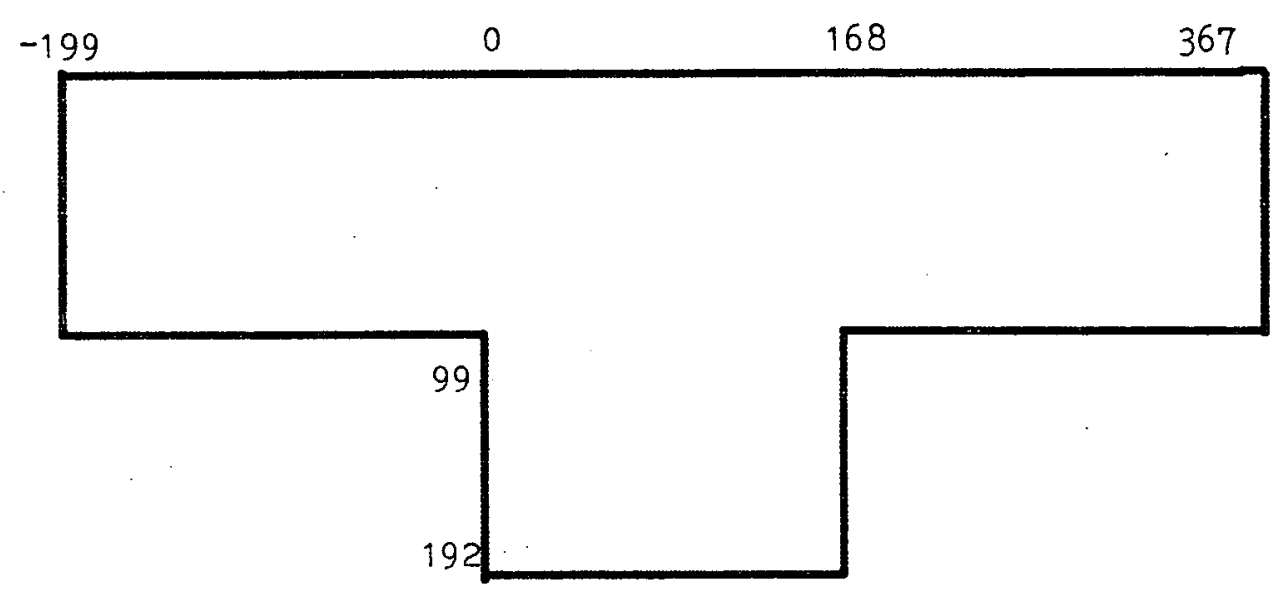

Figures 2.11 and 2.12 are snapshots at time 200 millisecondes and 220 millisecondes respectively. In both we still observe the diffraction phenomena. Figure 2.13 represents the corresponding seismogram, depicting horizontal displacement. At time around 275 milliseconds there appears the same sort of disturbance as seen in figure 2.8, only here at a later time as the corner is further from the source. We remark that an analysis of the velocity indicates that the nature of the diffracted disturbance is probably an $S$ wave; and again we emphasize that the amplication factor in the seismograms at later times is quite large. 


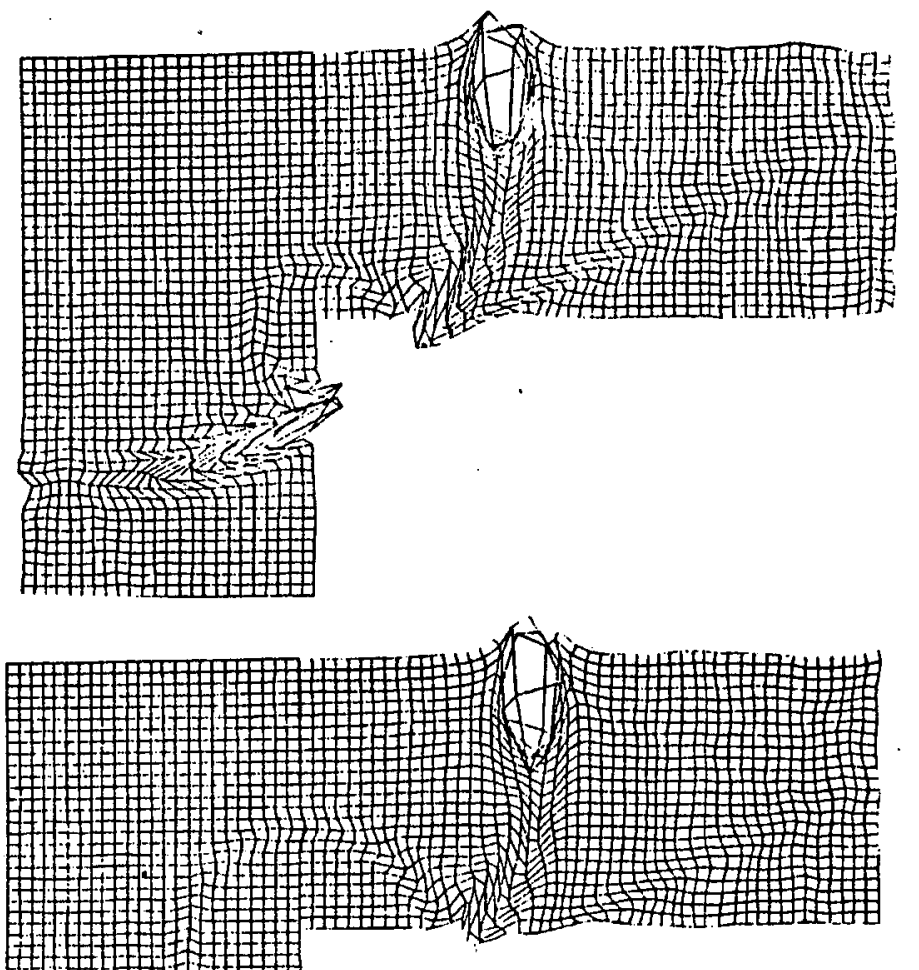

Figure 2.11:

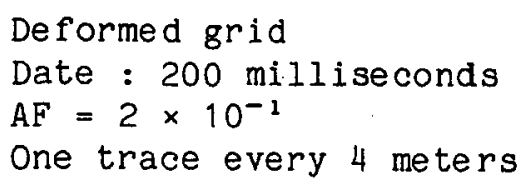

Figure 2.12:

Deformed grid

Date : 220 milliseconds

$\mathrm{AF}=2 \times 10^{-1}$

One trace every 4 meters
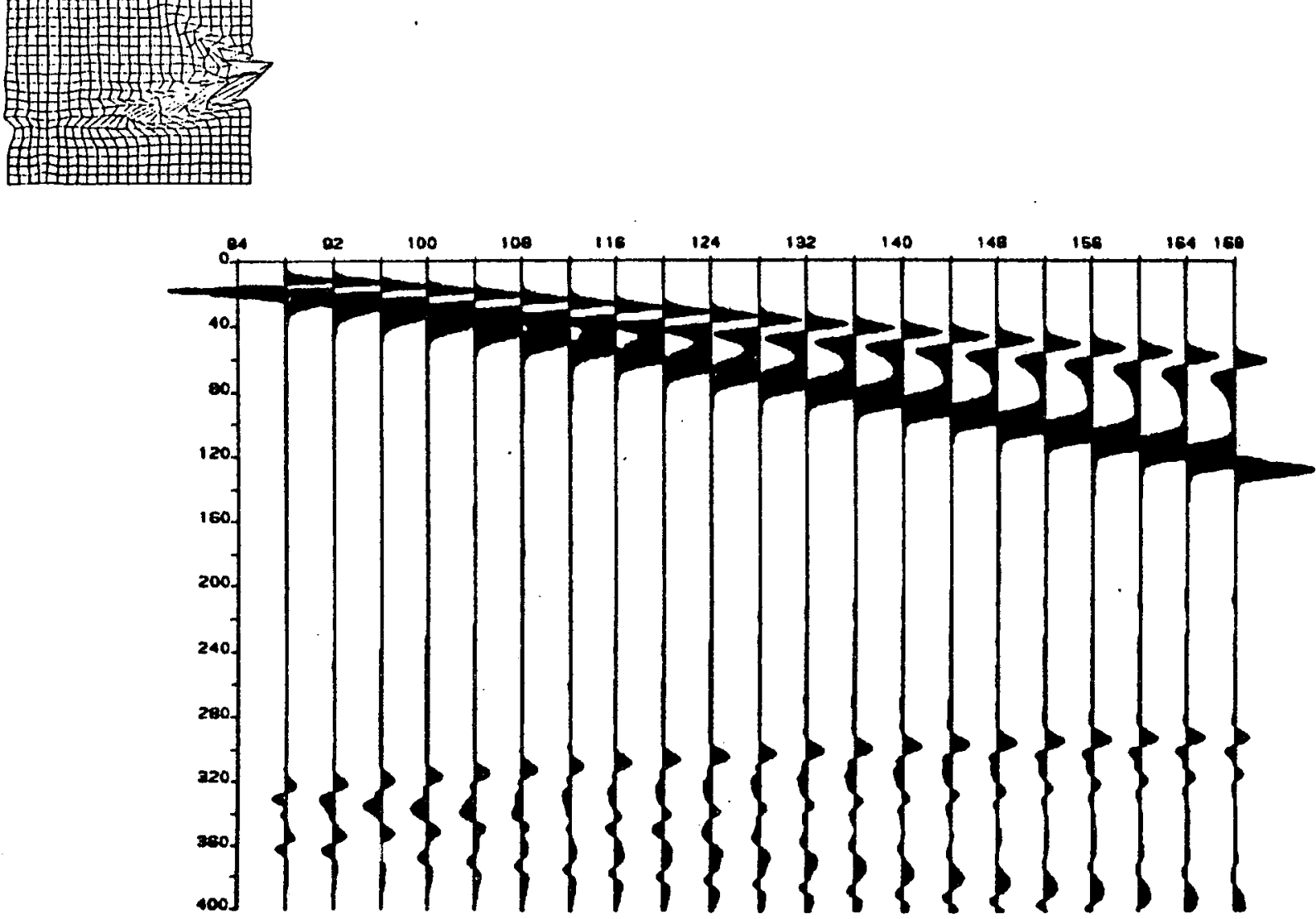

Figure 2.13: Horizontal Displacement

$$
\begin{aligned}
& \text { IFA }=1 \times 10^{-2} \\
& G A=1 \times 10^{-4}
\end{aligned}
$$




\section{CONCLUSION}

The ears solution is effective in eliminating the reflection and conversion of the Rayleigh wave at the artificial absorbing boundary. It also eliminates the parasitic Rayleigh wave generated when the incident $P$ wave reaches the corner joining the free boundary with the artificial boundary. However, the interior corners introduced by the addition of the ears do give rise to a diffraction that, though smaller in amplitude than the parasitic Rayleigh waves, is still of an order that can be detected in the snapshots and seismograms. We can hope to improve these results by modifying the absorbing condition in a neighborhood of the corner.

Finally, one should note that though the addition of the ears should not significantly increase the time of calculation, it does complicate the programming. Still this complication is not extrordinary as the ears may be programmed once and for all independently of the complexity of the model. 


\section{III - A CONDITION TRANSPARENT FOR P, $S$, AND R WAVES}

\section{III- 1 -Derivation and analysis of a new condition}

We restrict our attention to the case of a half plane $\Omega$ with boundary the line $\Gamma$ having unit normal vector $n$. The first order condition

$$
\mathscr{L}_{1}(u)=\sigma \cdot n+M \frac{\partial u}{\partial t}=0,
$$

with $M$ the symmetric positive definite matrix

$$
M=\left(\begin{array}{cc}
n_{1} & n_{2} \\
n_{2} & -n_{1}
\end{array}\right)\left(\begin{array}{cc}
\rho V_{P} & 0 \\
& \rho V_{S}
\end{array}\right)\left(\begin{array}{cc}
n_{1} & n_{2} \\
& \\
n_{2} & -n_{1}
\end{array}\right) \text {, }
$$

is transparent for all $P$ and $S$ waves with normal incidence. Let us now consider the differential operator

$$
\frac{\partial}{\partial t}+C n \cdot \operatorname{grad}
$$

where $C$ designates a positive velocity. Clearly we have

$$
\frac{\partial u}{\partial t}+C n \cdot \operatorname{grad} u=0
$$

for any $C$ wave with normal incidence, i.e. for any wave $u$ of the form

$$
u(x, t)=u_{C}(x \cdot \tau, x \cdot n-C t)
$$

where $\tau$ is a unit vector tangent to $u_{C}$ is an arbitrary vectorial function of two scalar variables. We introduce the operator :

$$
\mathscr{L}_{C}(u)=\left(\frac{\partial}{\partial t}+C n \cdot \operatorname{grad}\right) \mathscr{L}_{\mu_{1}}(u)
$$


and the boundary condition

$$
\mathscr{L}_{C}(u)=0
$$

on $\Gamma$.

We remark that this condition is :

- in the case of a left half plane with $\Gamma$ a vertical boundary

$$
\left(\frac{\partial}{\partial t}+c \frac{\partial}{\partial x_{1}}\right) \mathscr{L}_{1}(u)=0 \text {, on } \Gamma \text {, }
$$

with

$$
\mathscr{L}_{1}(u)=\left(\begin{array}{c}
\sigma_{11} \\
\sigma_{21}
\end{array}\right)+\rho \cdot\left(\begin{array}{cc}
v_{p} & 0 \\
0 & v_{S}
\end{array}\right)\left(\begin{array}{l}
\frac{\partial u_{1}}{\partial t} \\
\frac{\partial u_{2}}{\partial t}
\end{array}\right)
$$

- in the case of a lower half plane with $\Gamma$ a horizontal boundary

$$
\left(\frac{\partial}{\partial t}+c \frac{\partial}{\partial x_{2}}\right) \mathscr{L}_{1}(u)=0 \text {, on } r \text {, }
$$

with

$$
\mathscr{L}_{\mu_{1}}(u)=\left(\begin{array}{c}
\sigma_{12} \\
\sigma_{22}
\end{array}\right)+\rho\left(\begin{array}{ll}
v_{S} & 0 \\
0 & v_{p}
\end{array}\right)\left(\begin{array}{l}
\frac{\partial u_{1}}{\partial t} \\
\frac{\partial u_{2}}{\partial t}
\end{array}\right) .
$$

\section{Theorem 3.1}

The boundary condition 3.1 is transparent for :

- $P$ waves at normal incidence

- $S$ waves at normal incidence

- $C$ waves at normal incidence. 


\section{Proof :}

The first two points are clear. The last is also with the observation that $\mathscr{L}_{C}(u)$ may also be written in the form

$$
\mathscr{L}_{C}(u)=\mathscr{L}_{1}\left(\frac{\partial u}{\partial t}+c n \cdot \operatorname{grad} u\right)
$$

The operator $\frac{\partial}{\partial t}+c n \cdot g r a d$ is diagonal and thus as $c$ is constant commute with $\mathscr{L a}_{1}$

口

We turn now to the question of the stability of the condition 3.1. For simplicity of exposition we consider the case $\Omega$ the left half plane,

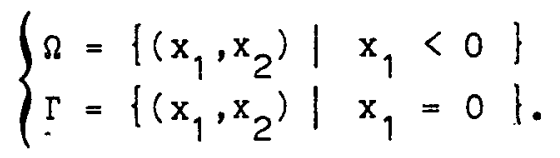

Then the problem may be written

$$
3.2 \begin{cases}\rho \frac{\partial^{2} u_{1}}{\partial t^{2}}=\frac{\partial}{\partial x_{1}} \sigma_{11}+\frac{\partial}{\partial x_{2}} \sigma_{12} \\ \frac{\partial^{2} u_{2}}{\partial t^{2}}=\frac{\partial}{\partial x_{1}} \sigma_{21}+\frac{\partial}{\partial x_{2}} \sigma_{22} & \text { in } \Omega, \\ \text { with boundary condition } & \text { on } \Gamma, \\ \mathscr{L}_{C}(u)=0 & \text { in } \Omega . \\ \text { and } w 1 t h \text { initial conditions } & \\ \frac{\partial u}{\partial t}(x, 0)=u^{0}(x) & \dot{u}^{\circ}(x)\end{cases}
$$


Theorem 3.2 :

The problem 3.2 is well posed.

\section{Proof :}

We introduce the function $w$,

$$
w=\frac{\partial u}{\partial t}+c \frac{\partial u}{\partial x_{1}}
$$

As $u$ satisfies the equations of elastodynamics in $\Omega$, so does $w$. Also $w$ satisfies the initial conditions

$$
\begin{cases}w(x, 0)=w^{0}(x)=\dot{u}^{0}(x)+c \frac{\partial u^{0}}{\partial x_{1}}(x) & \text { in } \Omega . \\ \frac{\partial w}{\partial t}(x, 0)=\dot{w}^{0}(x)=\frac{\partial^{2} u}{\partial t^{2}}(x, 0)+c \frac{\partial^{2} u}{\partial t \partial x_{1}}(x, 0) & \end{cases}
$$

An expression for $\frac{\partial^{2} u}{\partial t^{2}}(x, 0)$ may be deduced from the equations satisfied by $u$ at the initial time $t=0$. Furthermore, the function $w$ satisfies the boundary condition

$$
\mathscr{L} \mathscr{L}_{1}(w)=0, \quad \text { on } \Gamma \text {. }
$$

We know (cf. $\mathbf{S}_{1}$ ) that one can obtain energy estimations for w. Thus we deduce estimations for $u$ by integrating the equation

$$
\left\{\begin{array}{l}
\frac{\partial u}{\partial t}+c \frac{\partial u}{\partial x_{1}}=w \\
u(x, 0)=u^{0}(x),
\end{array}\right.
$$

and the theorem follows.

口

It is easy to check that in the operator $\mathscr{L A}_{\mathrm{c}}$, second derivatives in the normal direction to the boundary exist, which causes troubles : 
$-24-$

The expression for the boundary condition 3.1 may be transformed in such a way that only first order derivatives in the direction normal to $\Gamma$ appear. In the expression for $\mathscr{L}_{C}(u)$,

$$
\mathscr{L}_{C}(u)=\frac{\partial}{\partial t}(\sigma \cdot n)+C n \cdot \operatorname{grad}(\sigma \cdot n)+M\left(\frac{\partial^{2} u}{\partial t^{2}}+C \frac{\partial}{\partial t} n \cdot \operatorname{grad} u\right),
$$

the term containing the second order normal derivative is $\mathrm{Cn} \cdot \operatorname{grad}(\sigma \cdot \mathrm{n})$. Assuming that the equations of elastodynamics are satisfied on $\Gamma$, we deduce that

$$
n \cdot \operatorname{grad}(\sigma \cdot n)=-\tau \cdot \operatorname{grad}(\sigma \cdot \tau)+\rho \frac{\partial^{2} u}{\partial t^{2}}
$$

where $\tau$ is a unit vector tangent to $\Gamma$.

Combining these two equations we obtain

$$
\left\{\begin{array}{c}
\frac{\partial}{\partial t}(\sigma \cdot n)-C \tau \cdot \operatorname{grad}(\sigma \cdot \tau)+(M+\rho C 1) \frac{\partial^{2} u}{\partial t_{2}} \\
+C M \frac{\partial}{\partial t} n \cdot \operatorname{grad} u=0 .
\end{array}\right.
$$

Put

$$
\left\{\begin{array}{l}
\tilde{\mathscr{L}}_{C}(u)=\frac{\partial}{\partial t}(\sigma \cdot n)-C(\tau) \operatorname{grad}(\sigma \cdot \tau)+(M+-C 1) \cdot \frac{\partial^{2} u}{\partial t^{2}} . \\
+C M \frac{\partial}{\partial t} n \cdot \operatorname{grad} u=0 .
\end{array}\right.
$$

The operator $\tilde{\mathscr{L}}_{\mathrm{C}}(u)$ may be written :

- in the case of a left half plane with $\Gamma$ a vertical boundary

$$
\mathscr{L}_{C}(u)=\frac{\partial}{\partial t}\left(\begin{array}{c}
\sigma_{11} \\
\sigma_{21}
\end{array}\right)-c \frac{\partial}{\partial x_{2}}\left(\begin{array}{c}
\sigma_{12} \\
\sigma_{22}
\end{array}\right)+\rho\left(\begin{array}{cc}
v_{P}+C & 0 \\
0 & v_{S}+C
\end{array}\right) \frac{\partial^{2} u}{\partial t^{2}}+\rho\left(\begin{array}{cc}
C v_{p} & 0 \\
0 & C v_{S}
\end{array}\right) \frac{\partial^{2} u}{\partial t \partial x_{1}}
$$


- in the case of a lower half plane with $\Gamma$ a horizontal boundary

$$
\left\{\begin{array}{c}
\tilde{\mathscr{L}}_{C}(u)=\frac{\partial}{\partial t}\left(\begin{array}{l}
\sigma_{12} \\
\sigma_{22}
\end{array}\right)-c \frac{\partial}{\partial x_{1}}\left(\begin{array}{c}
\sigma_{11} \\
\sigma_{21}
\end{array}\right)+\rho\left(\begin{array}{cc}
v_{S}+C & 0 \\
0 & v_{P}+C
\end{array}\right) \frac{\partial^{2} u}{\partial t^{2}}+ \\
+\rho C\left(\begin{array}{ll}
v_{S} & 0 \\
0 & v_{P}
\end{array}\right) \frac{\partial^{2} u}{\partial t \partial x_{2}}
\end{array}\right.
$$

\section{Theorem 3.3:}

The boundary condition 3.1 is equivalent to the condition

$$
\tilde{\mathscr{L}}_{C}(u)=0
$$

\section{III- 2 - Plane wave analysis}

As in the case of the first order condition, the condition 3.1 may be subjected to analysis in terms of the reflection coefficients $\mathrm{R}_{\mathrm{PP}}, \mathrm{R}_{\mathrm{PS}}, \mathrm{R}_{\mathrm{SS}}$, and $\mathrm{R}_{\mathrm{SP}}$, defined in paragraph 1.2 .

We take for domain $\Omega$ the left half plane $\left\{x: x_{1}<0\right\}$ and assume that on the boundary $\Gamma_{2} \quad\left\{x: x_{1}=0\right\}$ we have the absorbing boundary condition 3.1

$$
\left\{\begin{array}{l}
\mathscr{L}_{\mathrm{C}}(u)=\left(\frac{\partial}{\partial \mathrm{t}}+\mathrm{c} \frac{\partial}{\partial \mathrm{x}_{1}}\right) \quad{ }_{1}(u)=0 \\
\mathscr{L}_{\rho_{1}}(u)=\left(\begin{array}{l}
\sigma_{11} \\
\sigma_{21}
\end{array}\right)+\rho\left(\begin{array}{cc}
v_{\mathrm{P}} & 0 \\
0 & v_{\mathrm{S}}
\end{array}\right) \frac{\partial u}{\partial t} \cdot
\end{array}\right.
$$


Consider a harmonic $P$ wave of unit norm,

$$
u^{P}=u_{P} e^{i\left(k_{P} x-\omega t\right)}
$$

propagating toward the boundary $\Gamma_{2}$. Suppose that upon arriving at $\Gamma_{2}$, $u^{P}$ gives rise to a reflected $P$ wave $u^{P P}$ and a reflected $S$ wave $u^{P S}$,

$$
\left\{\begin{array}{l}
u^{P P}=R_{P P} u_{P P} e^{1\left(k_{P P} x-\omega t\right)} \\
u^{P S}=R_{P S} u_{P S} e^{1\left(k_{P S} x-\omega t\right)} .
\end{array}\right.
$$

If the angle of incidence (measured from the normal) of $u^{P}$ is $\theta$, then the angle of emergence of $u^{P P}$ is also $\theta$ and that of $u^{P S}$ is $\psi$, where $\psi$ is related to $\theta$ by snell's law

$$
\sin \psi=\frac{V_{S}}{V_{P}} \sin \theta .
$$

We know that the length of the propagation vectors, $k_{P}$ and $k_{P P}$, of the $P$ waves is $\frac{\omega}{V_{P}}$ and that the length of $k_{P S}$, the propagation vector of the $S$ wave, is $\frac{\omega}{V_{S}}$. 
Furthermore, the unit displacement vectors $u_{p}$ and $u_{p P}$ of the $P$ waves are parallel to their propagation vectors $k_{P}$ and $k_{P P}$ while the displacement vector $u_{P S}$ for the $S$ wave is perpendicular to $\mathrm{k}_{\mathrm{PS}}$

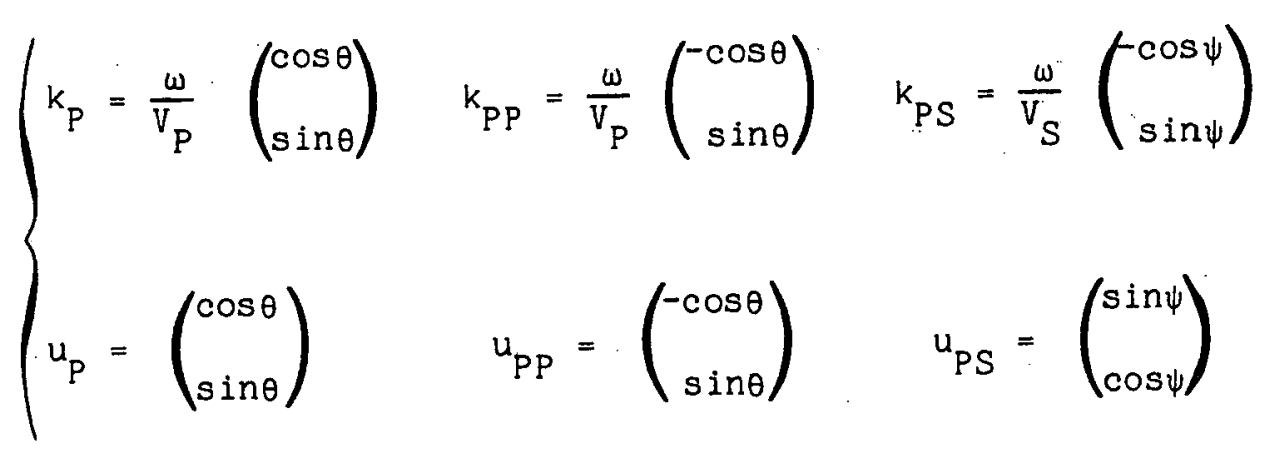

Now if we consider the sum $u$ of these three waves,

$$
u=u^{P}+u^{P P}+u^{P S} \text {, }
$$

we know that by construction $u$ satisfies the equations of elastodynamics in $\Omega$. By imposing that $u$ also satisfy the condition (3.1), on $\Gamma_{2}$, we obtain the following linear system in the unknowns $\mathrm{R}_{\mathrm{PP}}$ and $\mathrm{R}_{\mathrm{PS}}:$

$$
\left\{\begin{array}{l}
(\cos \theta+1)\left(2 \cos \theta+k^{2}-2\right)(m \cos \theta+k) R_{P P} \\
-k^{2} \sin \psi(2 \cos \psi+k)(m \cos \psi+1) R_{P S} \\
=(\cos \theta-1)\left(2 \cos \theta-k^{2}+2\right)(m \cos \theta-k) \\
\sin \theta(2 \cos \theta+k)(m \cos \theta+k) R_{P P} \\
+k^{2}(\cos \psi+1)(2 \cos \psi-1)(m \cos \psi+1) R_{P S} \\
=-\sin \theta(2 \cos \theta-k)(m \cos \theta-k),
\end{array}\right.
$$

where $\kappa$ is the ratio $\frac{V_{P}}{V_{S}}$ given as a function $v$ by

$$
K=\left(\frac{1(1-v)}{1-2 v}\right)^{1 / 2}
$$

and $m$ is the ratio $\frac{C}{V_{S}}$. 
Similarly we obtain a system in the unknowns. $R_{S P}$ and $R_{S S}$ by considering the reflection of a harmonic $S$ wave of unit amplitude

$$
u^{S}=u_{S} e^{i\left(k_{S} x-\omega t\right)}
$$

by the boundary $\Gamma_{2}$. For an angle of incidence $\theta$, there are a reflected $P$ wave $u^{S P}$ and a reflected $S$ wave $u^{S S}$.

$$
\begin{aligned}
& u^{S P}=R_{S P} u_{S P} e^{i\left(k_{S P} x-\omega t\right)} \\
& u^{S S}=R_{S S} u_{S S} e^{i\left(k_{S S} x-\omega t\right)}
\end{aligned}
$$

emerging at angles $\psi$ and $\theta$ respectively, with

$$
\text { (3.4) } \quad \sin \psi=\frac{V_{P}}{V_{S}} \sin \theta
$$

As before, the length of the propagation vector $k_{S P}$ of the $P$ wave is $\frac{\omega}{V_{P}}$, while that of $k_{S}$ and $k_{S S}$ is $\frac{\omega}{V_{S}}$, 
and the init displacement vector $u_{S P}$ is parallel to $k_{S P}$ winile $u_{S}$ and $u_{S S}$ are orthogonal to $k_{S}$ and $k_{S S}$ respectively.

$$
\begin{cases}k_{S}=\frac{\omega}{v_{S}}\left(\begin{array}{c}
\cos \theta \\
\sin \theta
\end{array}\right) & k_{S P}=\frac{\omega}{v_{P}}\left(\begin{array}{c}
-\cos \psi \\
\sin \psi
\end{array}\right) \\
u_{S}=\left(\begin{array}{c}
-\sin \theta \\
\cos \theta
\end{array}\right) & k_{S S}=\frac{\omega}{v_{S}}\left(\begin{array}{c}
-\cos \theta \\
\sin \theta
\end{array}\right)\end{cases}
$$

Again, the linear system in $R_{S P}$ and $R_{S S}$ is obtained by requiring that the sum $u$ of the incident wave $u^{S}$, with the two reflected waves $u^{S P}$ and $u^{S S}$ satisfy the boundary condition 3.1 on $\Gamma_{2}$. We have thus

$$
\left\{\begin{array}{l}
(\cos \psi+1)\left(2 \cos \psi+k^{2}-2\right)(m \cos \psi+k) R_{S P} \\
-K^{2} \sin \theta(2 \cos \theta+k)(m \cos \theta+1) R_{S S} \\
=-k^{2} \sin \theta(2 \cos \theta-k)(m \cos \theta-1) \\
\sin \psi(2 \cos \psi+k)(m \cos \psi+k) R_{S P} \\
+k^{2}(\cos \theta+1)(2 \cos \theta-1)(m \cos \theta+1) R_{S S} \\
=-K^{2}(\cos \theta-1)(2 \cos \theta+1)(m \cos \theta-1)
\end{array}\right.
$$

We remark that equation 3.4 implies that for $\theta$ larger than $\arcsin \frac{1}{K}, \sin \psi$ is larger than $1, i . e, \theta$ is no longer real. In this case, the propagation vector $k_{S P}$ becomes :

$$
k_{S P}=\frac{\omega}{V_{S}}\left(\begin{array}{ll}
-i & \sin ^{2} \psi-k^{-2} \\
\sin \psi
\end{array}\right),
$$

and the reflected $P$ wave

$$
u^{S P}=R_{S P} u_{S P} e^{\frac{\omega}{v_{S}} \cdot\left(\sin ^{2} \psi-k^{-2}\right) x_{1}} e^{i\left(\frac{\omega}{V_{S}} \sin \psi x_{2}-\omega t\right)}
$$


is no longer a harmonic wave but is a surface wave propagating along the boundary $\Gamma_{2}$ with amplitude decreasing exponentiaily with distance from $\Gamma_{2}$.

Theorem 3.3 The reflection coefficients $R_{P P}, R_{P S}, R_{S P}$, and $\mathrm{R}_{\mathrm{SS}}$ depend only on the angle of incidence $\theta$, the poisson coefficient $v$, and the ratio $c / V_{S}$, and for small angles of incidence $\theta$ :

$$
\begin{aligned}
- \text { for } \mathbf{C}=\mathbf{V}_{\mathrm{P}-} \\
\mathrm{R}_{\mathrm{PP}}(\theta, \nu)=O\left(\theta^{4}\right) \\
\mathrm{R}_{\mathrm{PS}}(\theta, \nu)=O\left(\theta^{3}\right) \\
\mathrm{R}_{\mathrm{SS}}(\theta, \nu)=O\left(\theta^{2}\right) \\
\mathrm{R}_{\mathrm{SP}}(\theta, \nu)=O(\theta)
\end{aligned}
$$

- for $C=V_{S-}$

$$
\begin{aligned}
& R_{P P}(\theta, \nu)=O\left(\theta^{2}\right) \\
& R_{P S}(\theta, v)=O(\theta) \\
& R_{S S}(\theta, \nu)=O\left(\theta^{4}\right) \\
& R_{S P}(\theta, \nu)=O\left(\theta^{3}\right)
\end{aligned}
$$

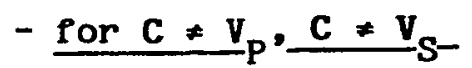

$$
\begin{aligned}
& R_{P P}\left(\theta, v, C / V_{S}\right)=O\left(\theta^{2}\right) \\
& R_{P S}\left(\theta, v, C / V_{S}\right)=O(\theta) \\
& R_{S S}\left(\theta, \nu, C / V_{S}\right)=O\left(\theta^{2}\right) \\
& R_{S P}\left(\theta, v, C / V_{S}\right)=O(\theta) .
\end{aligned}
$$


Proof :

Using 3.3 , a direct calculation yields that :

$$
\left\{\begin{array}{l}
R_{P P}=\left[-\frac{\left(4-k^{2}\right)(1+k) m}{4 k^{3}(m+k)}+O\left(\theta^{2}\right)\right]\left(1-\frac{k}{m}-\frac{\theta^{2}}{2}\right) \theta^{2} \\
R_{P S}=\left[-\frac{(2-k) m}{2 k^{2}(m+1)}+O\left(\theta^{2}\right)\right]\left(1-\frac{k}{m}-\frac{\theta^{2}}{2}\right) \theta .
\end{array}\right.
$$

Thus for $C=V_{P}$ we have $m=k$ and the term $\left(1-\kappa / m-\theta^{2} / 2\right)$ gives another factor of $\theta^{2}$ in the expressions for $R_{P P}$ and $R_{P S} \cdot$

Similarly from 3.5 we obtain

$$
\left\{\begin{array}{l}
\mathrm{R}_{\mathrm{SS}}=\left[-\frac{(k-4)(k+1) \mathrm{m}}{4 k(m+1)}+O\left(\theta^{2}\right)\right]\left(1-\frac{1}{m}-\frac{\theta^{2}}{2}\right) \theta^{2} \\
\mathrm{R}_{S P}=\left[-\frac{(2-k) m}{2(m+k)}+O\left(\theta^{2}\right)\right]\left(1-\frac{1}{m}-\frac{\theta^{2}}{2}\right) \theta
\end{array}\right.
$$

and observe that for $C=V_{S}$ a factor of $\theta^{2}$ is gained from the term $\left(1-1 / m-\theta^{2 / 2}\right)$ in the expressions for $R_{S S}$ and $R_{S P}$.

In figures 3.1 and 3.2 the amplitudes ${ }^{R}{ }_{P P}, R_{P S},{ }^{R}{ }_{S S}$, and $R_{S P}$ for the boundary conditions $\mathscr{L}_{1}(u)=0$ and $\mathscr{L}_{C}(u)=0$ with $c=v_{P}$ may be compared. These curves give the reflection coefficients for various values of the poisson coefficient $\nu$, from 0.0 to 0.48 , as functions of the angle of incidence $\theta$. We have not distinguished here the different values of $v$ : we want only to emphasize the weak influence of this parameter. We remark that even for the coefficients $R_{S P}$ and $R_{S S}$ which are of the same order in $\theta$ for both conditions, the results seem to be better for the condition. $\mathscr{L}_{C}(u)=0$.

The corresponding curves for the condition $\mathscr{L}_{C}(u)=0$ with $C=V_{S}$ and $C=V_{R}$ are shown in figure 3.2. We can easily see that, for all values of $C$ we have considered, the various reflection cefficients have been uniformily improved (not only for small values of $\theta$ ). 
First order
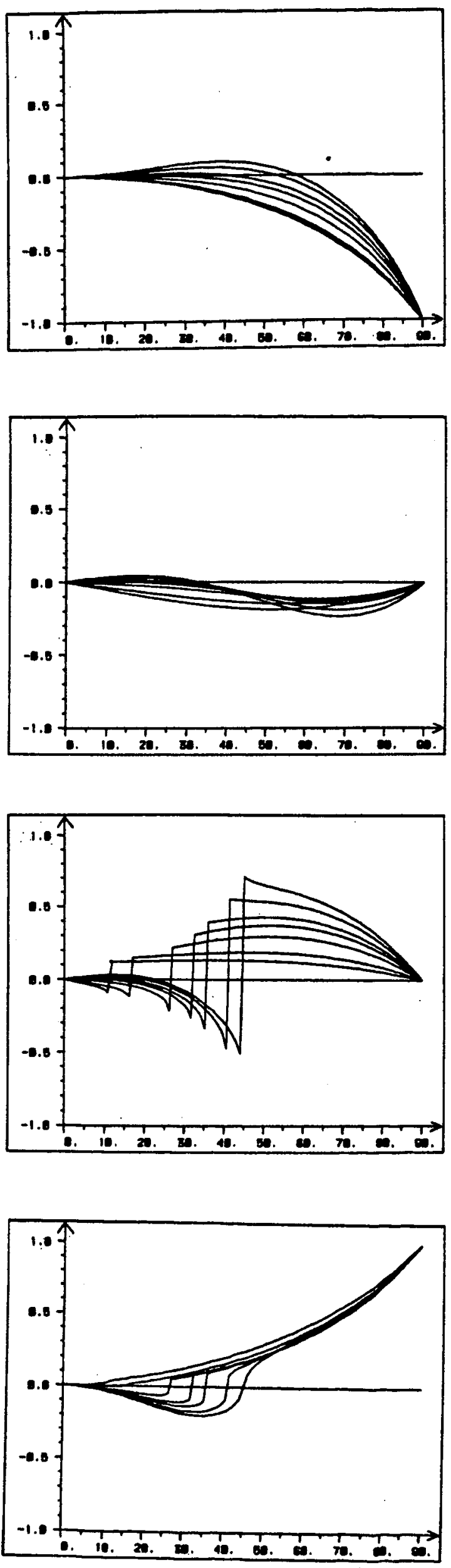

Figure 3.1 :

$$
\mathscr{L}_{\mathrm{C}} \mathrm{u}=0 \quad \mathrm{c}=\mathrm{v}_{\mathrm{P}}
$$
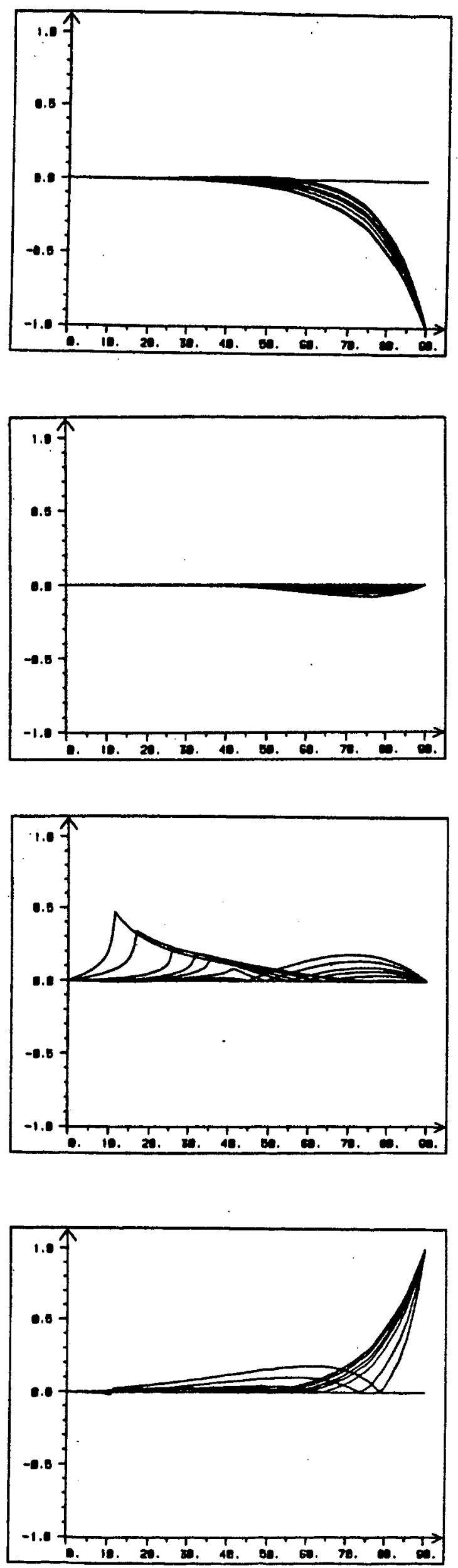

Reflection Coefficients 
$\mathrm{C}=\mathrm{v}_{\mathrm{S}}$
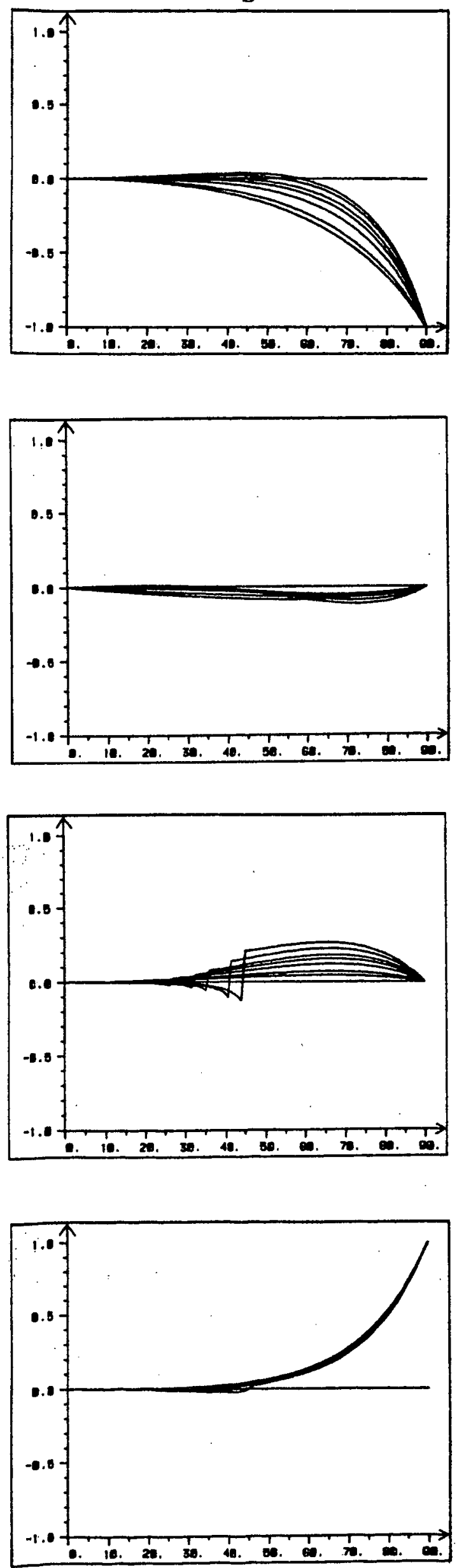

$C=C_{R}$
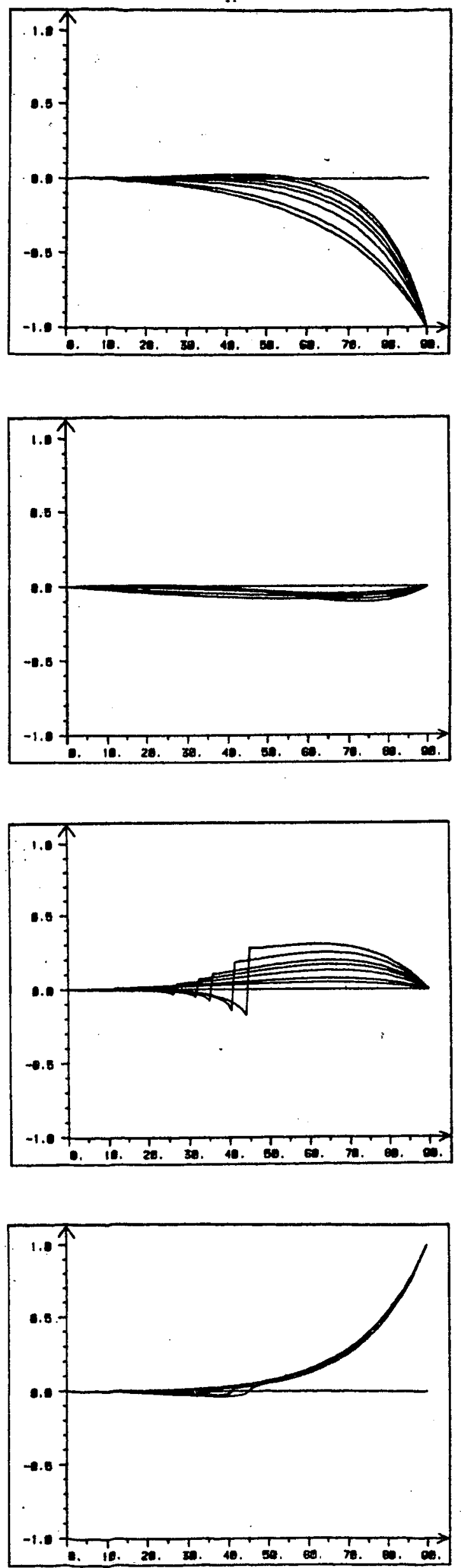

Figure 3.2: Reflection Ceofficients 


\section{III- 3 - A condition transparent for $P, S$ and $R$ waves}

We return now to our example of the half space $x_{2}<0$ with free boundary $\Gamma_{1}, x_{2}=0$. We restrict our domain of calculation, as in paragraph 1.2 , to the quarter plane

$$
\Omega=\left\{x_{1}: x_{1} \leqq 0 \text { and } x_{2} \leq 0\right\}
$$

and take for boundary condition on the artificial boundary $\Gamma_{2}, x_{1}=0$, the condition $3.1, \mathscr{L}_{\mathrm{VR}}(u)=0$.

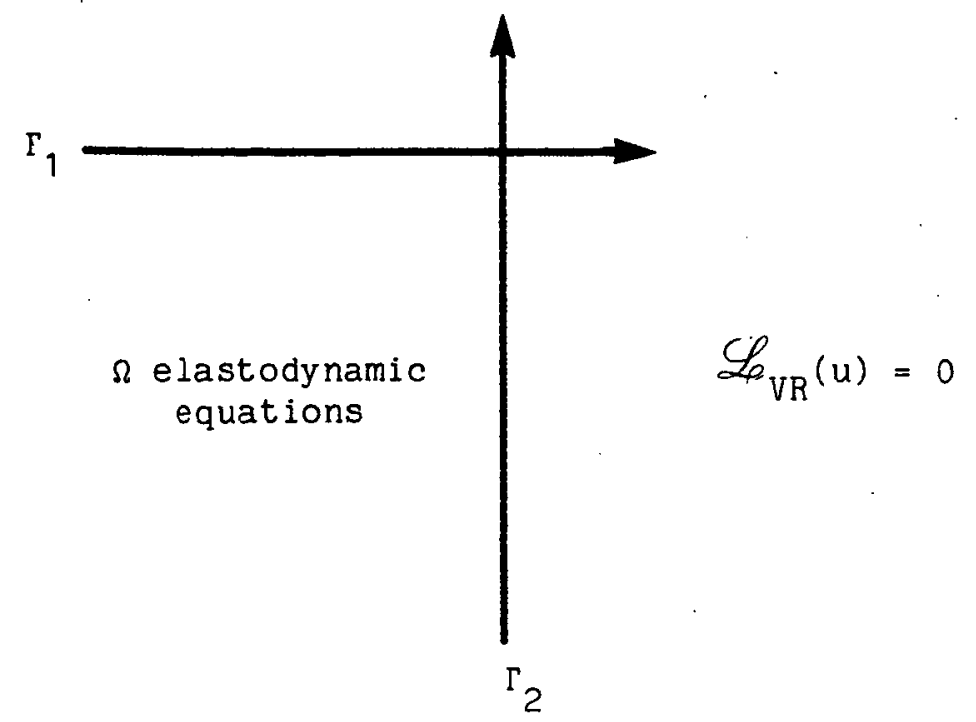

Thus all P, $S$ and Rayleigh waves at normal incidence are absorbed by the boundary $\Gamma_{2}$.

Remark : In order that Rayleigh waves traveling along the boundary $\Gamma_{1}$ be absorbed by the boundary $\Gamma_{2}$, we need to take $C=V_{R}$ the velocity of the Rayleigh wave. On the otherhand, theorem 3.3 indicates that it wculd also be of interest to take $C=V_{P}$ or $C=V_{S}$ in order to have a higher order absorbing condition for $P$ waves or for $S$ waves. Thus we are led to define $\mathrm{C}=\mathrm{C}\left(\mathrm{x}_{2}\right)$ to be a smooth function of $\mathrm{x}_{2}$, having value $V_{R}$ for small $x_{2}$ but having value say $V_{p}$ for large values of $x_{2}$ where the amplitude of the Rayleigh wave should be negligible though a rigorous analysis in this case remains to be done. 
This condition still, of course, needs to be tested numerically. There are at least two problems posed.

- The construction of a scheme for the discretization of the condition

- The treatment of the corner joining the free boundary and the absorbing boundary,

These we shall try to solve at a later date.

\section{CONCLUSION}

The geometric solution does not yield quite all of the results that one might hope for; however, some possibilities for improving it have been indicated. The $P-S-R$ condition appears to be a theoretical solution to the problem of the parasitic reflection of Rayleigh waves. This remains to be confirmed numerically a task which could pose some small difficulties. 


\section{REFERENCES}

Clayton,R. and Engquist, $B$.

"Absorbing Boudary Conditions for Acoustic and Elastic Wave Equations",

Bull. Seismol. Soc. Amer. 67(6), (1977), pp.1529-1540.

[2]

Engquist, B. and Majda, $A$., "Radiation Boundary Conditions for Acoustic and Elastic Wave Calculations",

Comm. Purc. Appl. Math. 32 (1979), pp.313-357.

[3].

Halpern, L.,

"Etude de conditions aux limites absorbantes pour des schémas numériques relatifs à des équations hyperboliques linéaires",

Thèse dé 3 ème Cycle, Université Paris VI, (1980).

[4] Lysmer, J. and Kuhlmeyer, R.L.,

"Finite dynamic model for infinite media",

J. Eng. Mech. Div, ASCE 95 EM4, (1969), pp.859-877. 
